\title{
COMPLEX INTERPOLATION OF NORMED AND QUASINORMED SPACES IN SEVERAL DIMENSIONS. II. PROPERTIES OF HARMONIC INTERPOLATION
}

\author{
ZBIGNIEW SLODKOWSKI
}

\begin{abstract}
This paper is a continuation of the study of harmonic interpolation families of normed or quasinormed spaces parametrized by points of a domain in $\mathbf{C}^{k}$. It is shown, among other things, that each of the following properties holds for all the intermediate quasinormed spaces, if it holds for all given boundary spaces: (1) being a normed space; (2) being a Hilbert space; (3) satisfying the triangle inequality by the $r$ th power of the quasinorm; (4) being uniformly convex; and (5) being uniformly smooth. As a principal tool, the notion of a harmonic set valued function (a generalization of analytic multifunction) is introduced and studied.
\end{abstract}

\section{INTRODUCTION}

This paper is a continuation of [18], where a variety of complex interpolation methods for families of normed or quasinormed spaces, parametrized by points of a domain in $\mathbf{C}^{k}$, was introduced and studied. These methods, denoted by $P_{Q}^{\mathrm{co}}$ and $P_{Q}^{\mathrm{psh}}$, where controlled by pseudoconvex classes $Q$, with local maximum properites, of functions on $\mathrm{C}^{k}$ (cf. $[18,(1.0)-(1.12)]$ ). In the natural, special case when $Q$ is the class of all subharmonic functions on $\mathbf{C}^{k}$, the corresponding interpolation method is called the harmonic interpolation method. Its basic properties, including existence, uniqueness, duality and reiteration, were obtained in $[18, \S 8]$ as special cases of the general results valid for all the $P_{Q}^{\text {co }}$ and $P_{Q}^{\mathrm{psh}}$-methods (with exception for the duality in the latter case). In this paper we restrict our attention to the harmonic interpolation method and establish its further properties.

A summary of basic results on the harmonic interpolation and some background can be found in [18, $\S \S 8$ and 1$]$. However, we review as needed necessary definitions and facts, and so the actual consulting of [18] is not required. ( $\S 6$ may constitute an exception.)

Recall first that a function $u(z, w)$, usc (=upper semicontinuous) on $U \subset$ $\mathbf{C}^{k} \times \mathbf{C}^{n}$, is said to be of class $P_{\text {subh }}$ if for every holomorphic map $f: G_{0} \rightarrow \mathbf{C}^{n}$,

Received by the editors March 7, 1988 and, in revised form, June 13, 1988.

1980 Mathematics Subject Classification (1985 Revision). Primary 46M35, 32F05; Secondary 30G30 46B20.

This research was supported in part by National Science Foundation grant DMS-870227. 
$G_{0} \subset \mathbf{C}^{k}$, the composition $z \rightarrow u(z, f(z))$ is subharmonic. Whenever we have a class $P$ of usc functions on open subsets of $\mathbf{C}^{N}$, we say that a locally closed set $X \subset \mathbf{C}^{N}$ has the local maximum property relative to $P$ (or that functions of class $P$ have local maximum property on $X$ ) if for every compact set $K \subset \mathbf{C}^{N}$, such that $X \cap K$ is compact, and for every function $u \in P$, defined near $K$, $\max u|X \cap K \leq \max u| X \cap(\partial K)$.

If $G \subset \mathbf{C}^{k}$ is a bounded domain, regular with respect to the Dirichlet problem, and $\left\{\mathbf{C}^{n}, p_{\zeta}(\cdot)\right\}_{\zeta \in \partial G}$ is a continuous family of (finite dimensional) quasinormed spaces, then there exists a unique continuous family of quasinormed spaces $\left\{\mathbf{C}^{n}, \tilde{p}_{z}(\cdot)\right\}_{z \in \bar{G}}$ (called the harmonic interpolation family), such that $\tilde{p}_{\zeta}=p_{\zeta}, \zeta \in \partial G$, and (1) the set $\left\{(z, w) \in G \times \mathbf{C}^{n}: \tilde{p}_{z}(w) \leq 1\right\}$ has local maximum property with respect to functions of class $P_{\text {subh }}$, and (2) the function $(z, w) \rightarrow \tilde{p}_{z}(w)$ belongs to $P_{\text {subh }}\left(G \times \mathbf{C}^{n}\right)$. In the normed case, closely related results were independently obtained by Coifman and Semmes and announced in [5] (see also Rochberg [10]).

The emphasis of this paper is on studying those geometric properties of normed or quasinormed spaces that must hold for the intermediate quasinorms $\tilde{p}_{z}, \quad z \in G$, provided they are satisfied by the given quasinorms $p_{\zeta}, \zeta \in \partial G$. In particular, we show in $\S 2$ that the interpolation of norms necessarily leads to norms. An analogous fact holds for $r$-norms for each $r \in(0,1)$ (where an $r$-norm is a quasinorm whose $r$ th power satisfies the triangle inequality). We give also a new, very simple proof of the fact, obtained first by Coifman and Semmes, that the (harmonic) interpolation of Hilbertian (=inner product) norms always produces Hilbertian norms (cf. Corollary 2.7). We developed this direct, interpolation-theoretic proof in response to a suggestion of Rochberg [10].

Various properties related to uniform convexity and smoothness of fiber spaces exhibit similar regularity with respect to the harmonic interpolation. If the squares of the boundary quasinorms $p_{\zeta}(w)$ have $L^{\infty}$ second order derivatives with respect to $w$, uniformly on $\partial G$, then the same is true for the intermediate quasinorms on $G$ (Theorem 2.12). In $\S 5$, estimates of moduli of convexity and smoothness of the intermediate quasinorms $\tilde{p}_{z}, z \in G$, in terms of those of $p_{\zeta}, \zeta \in \partial G$, are given. It is also shown, among other things, that various geometric constants characterizing the quasinormed spaces $\left(\mathbf{C}^{n}, \tilde{p}_{z}\right)$, $z \in G$, are sub- or superharmonic functions of the parameter $z$ (cf. Theorems 2.10 and 5.4 and Remark 5.5).

In proving the above-mentioned results, we use systematically harmonic multifunctions (cf. Definition 2.1). Similarly as analytic multifunctions, explored by the author in $[11,13,15,16]$, harmonic multifunctions are set-valued generalizations of analytic maps which are defined by a certain maximum property. Their properties are developed in $\S \S 2,3$ and 4 (but only to the extent required by the applications of this paper). In particular, we prove a result on the composition of an analytic multifunction with a harmonic one (Theorem 4.5). Using 
this, we show that, if $\left\{\mathbf{C}^{n}, N_{t}(\cdot)\right\}_{t \in\left(0, t_{0}\right)}$ is an interpolation family of (quasinormed) spaces in the Calderón sense, and $h: G \rightarrow\left(0, t_{0}\right), G \subset \mathbf{C}^{k}$, is a harmonic function, then $\left\{\mathbf{C}^{n}, N_{h(z)}(\cdot)\right\}_{z \in G}$ is a harmonic interpolation family (Theorem 4.1). This proves a conjecture of Rochberg [10].

Already this theorem allows one to obtain many specific harmonic interpolation families. In addition to this, explicit examples of harmonic interpolation families of $l_{p}$ spaces (also for $p<1$ ) are presented in $\S 3$.

The exposition of material outlined so far $(\S \S 1-5)$ is essentially self-contained. $\S 6$ (of which $\S \S 1-5$ are independent) may require referring to [18]. Its purpose is to provide a wider perspective in which the reasons for various properties of the harmonic interpolation method stand out more clearly. We show that some of these properties extend to those $P_{Q}^{\mathrm{co}}$ - or $P_{Q}^{\mathrm{psh}}$-interpolation methods of [18] for which the parametrizing class $Q$ of functions is closed with respect to addition, while some other properties hold when the dual class $Q^{d}$ is closed with respect to addition (cf. Terminology 1.1 below). It turns out that if both $Q$ and $Q^{d}$ have this property, then $Q=$ the class of all subharmonic functions with respect to some metric (and conversely).

We would like to point out that Example 6.7 (as well as Corollary 6.8 and Example 6.9) can be read independently of the rest of $\S 6$. The example shows that a compact set fibered over a sphere in $\mathbf{C}^{2}$, with convex fibers, may have a polynomial hull with nonconvex fibers.

\section{Preliminaries: properties of the class $P_{\text {subh }}$}

Class $P_{\text {subh }}$ is a special case of classes $P_{Q}$ studied in $[19, \S 4]$. We list here for further reference some of its properties. Below, $P_{\text {subh }}$ stands for the class of all $P_{\text {subh }}$ functions on subsets of $C^{k+n}$.

$$
P_{\text {subh }}+P_{\text {subh }} \subset P_{\text {subh }} \text {. }
$$

If $U_{1} \subset \mathbf{C}^{k} \times \mathbf{C}^{n}, U \subset \mathbf{C}^{k} \times \mathbf{C}^{m}, u \in P_{\text {subh }}(U)$, and $(z, w) \rightarrow$ $(z, F(z, w)): U_{1} \rightarrow U$ is a holomorphic map, then the function $u_{1}(z, w)=u(z, F(z, w))$ is of class $P_{\text {subh }}$ on $U_{1}$.

All plurisubharmonic functions on $\mathbf{C}^{k+n}$ belong to $P_{\text {subh }}$.

$$
\begin{gathered}
P_{\text {subh }} \subset P_{k-1} . \\
P_{\text {subh }}=P_{\text {subh }}^{\text {psh }} . \\
P_{\text {subh }} \subset \text { subh } \quad\left(\text { on } \mathbf{C}^{k+n}\right) .
\end{gathered}
$$

If $u>0, \log u \in P_{\text {subh }}$ and $r>0$, then $u^{r} \in P_{\text {subh }}$.

If $u \in P_{\text {subh }},-\infty \leq a<u<b$, and $\rho$ is a nondecreasing convex function on $(a, b)$, then $\rho \circ u \in P_{\text {subh }}$. 
Terminology 1.1. $P_{k-1}$ in (1.5) denotes the class of all $(k-1)$-plurisubharmonic functions on $\mathbf{C}^{k+n}$. Recall that a $C^{(2)}$-smooth function $u$ belongs to $P_{q}$ if its complex Hessian has no more than $q$ negative eigenvalues; we then write $u \in C^{(2)} P_{q}$ (cf. [7]). If $P$ is an arbitrary class of usc functions on $\mathbf{C}^{N}$, we say that a usc function $v$ lies in the dual class $P^{d}$ if for every $u \in P$ and for every compact set $K \subset \operatorname{Dom}(u) \cap \operatorname{Dom}(v), \max (u+v)|K \leq \max (u+v)| \partial K$. We can now define $P_{q}$ as the dual class to $C^{(2)} P_{N-q-1}$ (cf. [12, §1] or [7]).

The class $P_{\text {subh }}^{\text {psh }}$ in (1.6) consists of those functions $u(z, w)$ in $P_{\text {subh }}$ which have plurisubharmonic slices $w \rightarrow u(z, w), z \in \mathbf{C}^{k}$. (The interpolating quasinorms were defined in [18] with the help of $P_{\text {subh }}^{\text {psh }}$; according to (1.6), we can just use $P_{\text {subh }}$.)

Remark 1.2. It was shown in [19, Lemma 4.11] that a $C^{(2)}$-smooth function $u(z, w)$ lies in $P_{\text {subh }}$ if and only if for every point $(a, b) \in \operatorname{Dom} u$ and for every $\mathbf{C}$-linear map $S: \mathbf{C}^{k} \rightarrow \mathbf{C}^{n}$, the form

$$
z \rightarrow \operatorname{Hess}^{\mathrm{C}} u_{(a, b)}(z \oplus S z, z \oplus S z)
$$

has nonnegative trace.

If $u$ is strictly plurisubharmonic in $w$, an equivalent condition is

$$
\operatorname{tr}\left(H_{11}-H_{12} H_{22}^{-1} H_{21}\right) \geq 0,
$$

where $H_{11}, H_{12}, H_{21}, H_{22}$ are the blocks of the complex Hessian corresponding to $\bar{z} z, \bar{z} w, \bar{w} z, \bar{w} w$ (cf. [19, Lemma 4.11]).

Properties (1.1) through (1.3) follow immediately from the definition of $P_{\text {subh }}$. Property (1.7) is a consequence of the definition and of the similar property for subharmonic functions (cf. also [17, Proposition 1.14(iv)]). Property (1.7)(a) follows in the same way.

Proof of property (1.4). By the definition of $(k-1)$-plurisubharmonic functions [7; 14, §1; and 12, Proposition 1.1], we have to show: if $u \in P_{\text {subh }}(U), v(z, w)$ is a plurisubharmonic function defined in a neighborhood of a closed ball $\bar{B} \subset U$ and $L$ is a complex $k$-dimensional plane through the center $(a, b)$ of $B$, then

$$
(u+v)(a, b) \leq \max (u+v) \mid(\partial B) \cap L .
$$

Suppose this fails, then there is a neighborhood $V$ of $(\partial B) \cap L$ in $C^{k+n}$ such that

$$
(u+v)(a, b)>\sup (u+v) \mid \bar{V} .
$$

One can choose a $k$-dimensional complex plane $L_{1}$ through $(a, b)$ so that $L_{1} \cap \partial B \subset V$, and $L_{1}$ does not contain any direction parallel to $\{0\} \times \mathbf{C}^{n}$. Then $L_{1}$ is the graph of a $\mathbf{C}$-affine map $S: \mathbf{C}^{k} \rightarrow \mathbf{C}^{n}$. By $(1.8),(u+v)(a, b)>$ $\max (u+v) \mid L_{1} \cap \partial B$, which contradicts the local maximum property, and so also the subharmonicity of the function $z \rightarrow(u+v)(z, S(z))$. Q.E.D. 
Proof of property (1.5). Only the inclusion $P_{\text {subh }} \subset P_{\text {subh }}^{\text {psh }}$ requires the proof. Let $u \in P_{\text {subh }}(U), U \subset \mathbf{C}^{k+n}$. We have to show that for every complex line $L \subset \mathbf{C}^{n}$, the function $w \rightarrow u(z, w):(\{z\} \times L) \cap U \rightarrow[-\infty, \infty)$ is subharmonic. By (1.2), $u \mid\left(\mathbf{C}^{k} \times L\right) \cap U \in P_{\text {subh }}$, and so we can assume, without loss of generality, that $n=$ 1. Fix $(a, b) \in U \subset \mathbf{C}^{k} \times \mathbf{C}$. By the well-known criterion, it suffices to show that, whenever $h(w)$ is harmonic in $D$ and continuous on $\bar{D}$, where $D=D(b, r)$, $r>0$, is an open disc, then $u(a, b)+h(b) \leq \max \{u(a, w)+h(w): w \in \partial D\}$. Suppose this fails. By [14, Lemma 4.5], there is an $R$-affine function $l(w)$ and $b^{*} \in D$ such that

$$
v\left(a, b^{*}\right)=0>v(a, w), \quad w \in D \backslash\left\{b^{*}\right\},
$$

where $v(z, w)=u(z, w)+h(w)+l(w)$. By (1.1) and (1.3), $v(z, w)$ is of class $P_{\text {subh }}$ in a neighborhood of $(a, b)$. Define now $V\left(z, w_{1}, \ldots, w_{k}\right)=v\left(z, w_{1}\right)+$ $\cdots+v\left(z, w_{k}\right)$ in a neighborhood of the point $\left(a, b^{*}, \ldots, b^{*}\right) \in \mathbf{C}^{k} \times \mathbf{C}^{k}$. Near this point, $V$ is a $P_{\text {subh }}$ function (by properties (1.1) and (1.2)), and so a $P_{k-1}$ function, by (1.4). In particular, the slice function $w \rightarrow V(a, w)$ must have local maximum property. This contradicts (1.9) because

$$
V\left(a, w_{1}, \ldots, w_{k}\right)=v\left(a, w_{1}\right)+\cdots+v\left(a, w_{k}\right)<0=V\left(a, b^{*}, \ldots, b^{*}\right)
$$

if $w_{i} \neq b^{*}$ for some $i$. Q.E.D.

Proof of property (1.6). Let $u \in P_{\text {subh }}(U), U \subset \mathbf{C}^{k+n}$. By the last proof, $P_{\text {subh }}=$ $P_{\text {subh }}^{\text {psh }}$, and so $u(z, w)$ is subharmonic in $z$ and $w$ separately. Subharmonicity of such $u$ is obtained by proving the mean-value inequality for a sphere $S \subset$ $\mathbf{C}^{k+n}$, centered at $(a, b)$. If $\bar{B}$ is the projection of this sphere on $\mathbf{C}^{k}$, then $S=\bigcup_{z \in \bar{B}}\{z\} \times S_{z}$, where $S_{z} \subset \mathbf{C}^{n}$ are spheres in $\mathbf{C}^{n}$ centered at $b$. Then

$$
\begin{aligned}
\int_{S} u(z, w) d \sigma(z, w) & =\int_{\bar{B}} d v(z) \int_{S_{z}} u(z, w) d \sigma_{z}(w) \\
& \geq \int_{\bar{B}} d v(z) u(z, b) \geq u(a, b)
\end{aligned}
$$

where $d \sigma(z, w), d \sigma_{z}(w)$ and $d v(z)$ denote suitably normalized, surface and volume measures. Q.E.D.

Remark. Properties (1.4)-(1.6) can also be proved by using smooth approximation of functions of class $P_{\text {subh }}$ (or $C_{-\infty, 10 c}^{1,1}$ approximation, as in $\left.[19, \S 2]\right)$ and applying Remark 1.2 above.

Terminology. Throughout this paper "a quasinorm" will mean a continuous plurisubharmonic function $p: \mathbf{C}^{n} \rightarrow[0,+\infty)$ which is $\mathbf{C}$-homogeneous (in the sense that $p(\lambda z)=|\lambda| p(z)$ if $\left.\lambda \in \mathbf{C}, z \in \mathbf{C}^{n}\right)$ and positive on $\mathbf{C}^{n} \backslash\{0\}$. 


\section{HARMONIC MULTIFUNCTIONS AND REGULARITY PROPERTIES OF INTERMEDIATE QUASINORMS}

The following definition is analogous to that of the weak analytic multifunction, (cf. [11, Definition 4.1; 15, Definition 3.2]).

Definition 2.1. Let $z \rightarrow K(z): G \rightarrow 2^{\mathrm{C}^{n}}$, where $G \subset \mathbf{C}^{k}$ is open, be a usc (=upper semicontinuous) multifunction (=set-valued function) with compact sections $K(z), z \in G$. We say that $K(\cdot)$ is a harmonic multifunction if for every integer $s$ and for every function $u(z, \xi, w)$ of class $P_{\text {subh }}$ on an open set $H \subset G \times \mathbf{C}^{n+s}$ (where $z \in G, w \in \mathbf{C}^{n}, \xi \in \mathbf{C}^{S}$ ), the function $v(z, \xi)=$ $\max \{u(z, w, \xi): w \in K(z)\}$ is $P_{\text {subh }}$ on the set $\left\{(z, \xi) \in G \times \mathbf{C}^{s}:\{z\} \times K(z) \times\right.$ $\{\xi\} \subset H\}$.

Remark. If $s=0$, the condition requires that $v$ is a subharmonic function on $G$. In fact, this weaker condition characterizes harmonic multifunctions. (We omit the proof.)

The next fact is a special case of [18, Proposition 1.3]. (The proof in [18] was spelled out in the case when $H \supset \operatorname{gr}(K) \times \mathbf{C}^{S}$, but the required changes are trivial.)

Proposition 2.2. Let $z \rightarrow K(z): G \rightarrow 2^{\mathrm{C}^{n}}, G \subset \mathrm{C}^{k}$, be a usc multifunction with compact sections $K(z), z \in G$. Assume that the restrictions of functions of class $P_{\text {subh }}$ on $G \times \mathbf{C}^{n}$ to the graph of $K(\operatorname{gr}(K)=\{(z, w): z \in G, w \in K(z)\})$ have the local maximum property. Then $z \rightarrow K(z): G \rightarrow 2^{\mathrm{C}^{n}}$ is a harmonic multifunction.

It can be proved that the sufficient condition formulated in this proposition is also necessary.

The relation of harmonic multifunctions to harmonic functions is not very strong. In the case $a: U \rightarrow \mathbf{C}^{n}$ is a continuous single-valued mapping, then $K(z)=\{a(z)\}$ is a harmonic multifunction if and only if $a(\cdot)$ is an analytic mapping, and the same equivalence holds for analytic multifunctions. If $\varphi: U \rightarrow[-\infty,+\infty)$ is usc and $K(z)=\left\{w \in \mathbf{C}^{n}:|w| \leq e^{\varphi(z)}\right\}$, then $K(\cdot)$ is a harmonic multifunction if and only if $\varphi$ is a subharmonic function (and $K$ is analytic if and only if $\varphi$ is plurisubharmonic). The case of $\varphi$ harmonic would correspond to extremal harmonic multifunction with fixed boundary values $K(\zeta), \zeta \in \partial G$.

The following proposition is an analog of [11, Proposition 5.1].

Proposition 2.3. Let $z \rightarrow K(z): G \rightarrow 2^{\mathrm{C}^{n}}$ and $z \rightarrow L(z): G \rightarrow 2^{\mathrm{C}^{m}}$, where $G \subset \mathbf{C}^{k}$ is open, be harmonic multifunctions. Then

$$
z \rightarrow K(z) \times L(z): G \rightarrow 2^{\mathrm{C}^{n+m}}
$$

is also a harmonic multifunction.

Proof. Let $R(z)=K(z) \times L(z)$. We have to show that whenever $u\left(z, w_{1}, w_{2}, \xi\right)$ is a function of class $P_{\text {subh }}$ on an open set $H \subset G \times \mathbf{C}^{n+m+s}$, then the function 
$v(z, \xi)=\max \left\{u\left(z, w_{1}, w_{2}, \xi\right):\left(w_{1}, w_{2}\right) \in R(z)\right\}$ belongs to $P_{\text {subh }}\left(H_{0}\right)$, where $H_{0}=\left\{(z, \xi) \in G \times \mathbf{C}^{S}:\{z\} \times R(z) \times\{\xi\} \subset H\right\}$.

Let $u_{1}\left(z, w_{2}, \xi\right)=\max \left\{u\left(z, w_{1}, w_{2}, \xi\right): w_{1} \in K(z)\right\}$. Since $K_{1}(\cdot)$ is a harmonic multifunction, $u_{1}$ is a $P_{\text {subh }}$-function on $H_{1} \subset G \times \mathbf{C}^{m+s}$, where $H_{1}=\left\{\left(z, w_{2}, \xi\right):\{z\} \times K(z) \times\left\{\left(w_{2}, \xi\right)\right\} \subset H\right\}$. Observe now that $H_{0}=$ $\left\{(z, \xi):\{z\} \times L(z) \times\{\xi\} \subset H_{1}\right\}$ and $v(z, \xi)=\max \left\{u_{1}\left(z, w_{2}, \xi\right): w_{2} \in L(z)\right\}$. Since $L(\cdot)$ is a harmonic multifunction, $v \in P_{\text {subh }}\left(H_{0}\right)$. Q.E.D.

Our applications of harmonic multifunctions to harmonic interpolation rely on the next proposition and remark.

Proposition 2.4. Let $p_{z}: \mathbf{C}^{n} \rightarrow R, z \in G$, be quasinorms (as always, continuous, plurisubharmonic and complex-homogeneous) and denote $K(z)=\{w \in$ $\left.\mathbf{C}^{n}: p_{z}(w) \leq 1\right\}$. Then the following conditions are equivalent:

(i) $\operatorname{gr}(K)=\{(z, w): z \in G, w \in K(z)\}$ is a $P_{\text {subh-maximum set; }}$

(ii) $z \rightarrow K(z): G \rightarrow 2^{\mathrm{C}^{n}}$ is a harmonic multifunction;

(iii) spaces $\left\{\mathbf{C}^{n}, p_{z}(\cdot)\right\}_{z \in G}$ form a harmonic superinterpolation family (i.e. $P_{\text {subh }}^{\mathrm{psh}}$-superinterpolation family in the sense of [18, Definition 3.3]).

The concepts of sub- and superinterpolation were introduced by Rochberg [9] in the one-dimensional setting $(k=1)$. The several dimensional case was studied in $[18, \S 3.4]$.

We recall here that a family of quasinormed spaces $\left\{\mathbf{C}^{n}, p_{z}(\cdot)\right\}_{z \in G}$ is called a harmonic subinterpolation (superinterpolation) family if the function $(z, w) \rightarrow$ $p_{z}(w)$ is usc (lsc) and for every compact ball $\bar{B} \subset G$ and for every harmonic interpolation family on $\bar{B},\left\{\mathbf{C}^{n}, q_{z}(\cdot)\right\}_{z \in \bar{B}}$, such that $p_{\zeta}(w) \leq q_{\zeta}(w), \zeta \in \partial B$, $w \in \mathbf{C}^{n}\left(p_{\zeta}(w) \geq q_{\zeta}(w)\right)$, it holds that $p_{z}(w) \leq q_{z}(w), \quad z \in B, w \in \mathbf{C}^{n}$ $\left(p_{z}(w) \geq q_{z}(w), z \in B, w \in \mathbf{C}^{n}\right)$.

Spaces $\left\{\mathbf{C}^{n}, p_{z}\right\}_{z \in G}$ form a harmonic interpolation family if and only if they have both the subinterpolation and the superinterpolation properties.

Proof of Proposition 2.4. (i) $\Rightarrow$ (ii) by Proposition 2.2, and (i) and (iii) are equivalent by [18, Theorem 3.5]. It remains to prove that (ii) $\Rightarrow$ (iii). By [18, Remark 3.11 and Definition 3.3], we have to show that if $\bar{B} \subset G$ is a closed ball and $\left\{\mathbf{C}^{n}, q_{z}(\cdot)\right\}_{z \in \bar{B}}$ is a harmonic interpolation family such that

$$
p_{\zeta}(w) \geq q_{\zeta}(w), \quad \zeta \in \partial B, w \in \mathbf{C}^{n},
$$

then

$$
p_{z}(w) \geq q_{z}(w), \quad z \in B, w \in \mathbf{C}^{n} .
$$

By [18, Theorem 4.1] or the next remark, $(z, w) \rightarrow q_{z}(w)$ is a $P_{\text {subh }}$-function on $B \times \mathbf{C}^{n}$, and by Definition 2.1 the function $v(z)=\max \left\{q_{z}(w): w \in K(z)\right\}$ is subharmonic in $B$ (and clearly usc on $\bar{B}$ ). By $(2.1), v(\zeta) \leq 1$ for $\zeta \in \partial B$, and so $v(z) \leq \max v \mid \partial B \leq 1$. This implies (2.2). Q.E.D. 
Remark 2.5. (a) Quasinormed spaces $\left\{\mathbf{C}^{n}, p_{z}(\cdot)\right\}_{z \in G}$ form a harmonic interpolation family if they form both a (harmonic) super- and subinterpolation family.

(b) $\left\{\mathbf{C}^{n}, p_{z}(\cdot)\right\}_{z \in G}$ form a harmonic subinterpolation family if and only if the function $(z, w) \rightarrow \log p_{z}(w): G \times \mathbf{C}^{n} \rightarrow[-\infty,+\infty)$ is of class $P_{\text {subh }}$ (cf. [18, Remark 3.4 and Theorem 4.1]).

Further results on harmonic multifunctions are given in $\S \S 3$ and 4 . We will now discuss the applications which will be easily derived from the following, rather technical, lemma.

Lemma 2.6. Let $G \subset \mathbf{C}^{k}$ be a bounded open set and let quasinormed spaces $\left\{\mathbf{C}^{n}, p_{z}(\cdot)\right\}_{z \in \bar{G}}$ form a harmonic interpolation family. (Continuity on $\bar{G}$ is also assumed.) Let $a, b, c, d, r$ be positive real numbers. Assume that for every $z \in \partial G$ and $x, y \in \mathbf{C}^{n}$

$$
a p_{z}(x+y)^{r}+b p_{z}(x-y)^{r} \leq c p_{z}(x)^{r}+d p_{z}(y)^{r} .
$$

Then this inequality holds for every $z \in G$ (with the same constants).

Proof. By continuity, it suffices to prove (2.3) for $x, y \neq 0$. To prove it for specific $z_{0} \in G, x_{0}, y_{0} \in \mathbf{C}^{n} \backslash\{0\}$, let $\alpha=p_{z_{0}}\left(x_{0}\right), \beta=p_{z_{0}}\left(y_{0}\right)$, and replace $x_{0}$ by $\alpha x$ and $y_{0}$ by $\beta y$ in (2.3), where $p_{z_{0}}(x)=1=p_{z_{0}}(y)$. By this, it is enough to show that

$$
a p_{z}(\alpha x+\beta y)^{r}+b p_{z}(\alpha x-\beta y)^{r} \leq c \alpha^{r}+d \beta^{r}
$$

for every $x, y \in \mathbf{C}^{n}$ such that $p_{z}(x) \leq 1, p_{z}(y) \leq 1$, and arbitrary $\alpha, \beta>0$. Now let $u(z, x, y)=a p_{z}(\alpha x+\beta y)^{r}+b p_{z}(\alpha x-\beta y)^{r}$, with $\alpha, \beta$ fixed. By Remark 2.5(b) and property (1.2), functions $(z, x, y) \rightarrow \log p_{z}(\alpha x+\beta y)$ and $(z, x, y) \rightarrow \log p_{z}(\alpha x-\beta y)$ belong to $P_{\text {subh }}\left(G \times \mathbf{C}^{n} \times \mathbf{C}^{n}\right)$; applying properties (1.8) and (1.9), we conclude that $u \in P_{\text {subh }}\left(G \times \mathbf{C}^{2 n}\right)$. Denote by $W(z)$, $z \in G$, the unit ball of the quasinorm $p_{z}(\cdot)$. By Proposition 2.4, $z \rightarrow W(z)$ is a harmonic multifunction, and by Proposition 2.3 the product multifunction $z \rightarrow W(z) \times W(z): G \rightarrow 2^{\mathrm{C}^{2 n}}$ is harmonic. Hence, the function $v(z)=$ $\max \{u(z, x, y): x, y \in W(z)\}$ is subharmonic in $G$ (and usc in $G$ ).

By the construction of the function $v(\cdot)$, the condition (2.4) holds for a given quasinorm $p_{z_{0}}(\cdot)$ if and only if $v\left(z_{0}\right) \leq c \alpha^{r}+d \beta^{r}$; by the assumptions, it holds on $\partial G$, and so, by the subharmonicity of $v$, we get $v(z) \leq$ $\max \{v(\zeta): \zeta \in \partial G\} \leq c \alpha^{r}+d \beta^{r}$ for all $z \in \bar{G}$. Thus (2.4), and so (2.3), holds everywhere on $\bar{G}$. Q.E.D.

Corollary 2.7. Let $\left\{\mathbf{C}^{n}, p_{z}(\cdot)\right\}_{z \in \bar{G}}$ (where $G \subset \mathbf{C}^{k}$ is bounded and open) be a harmonic interpolation family of quasinormed spaces on $G$ and continuous on $\bar{G}$. Then

(a) if all the boundary quasinorms $p_{\zeta}(\cdot), \zeta \in \partial G$, are actually norms, then all the intermediate quasinorms $p_{z}(\cdot), z \in G$, are also norms;

(b) if all the boundary norms $p_{\zeta}, \zeta \in \partial G$, are Hilbertian, then all the intermediate ones are Hilbertian as well. 
Proof. (a) By the assumption, $p_{\zeta}(x+y) \leq p_{\zeta}(x)+p_{\zeta}(y), \zeta \in \partial G, x, y \in \mathbf{C}^{n}$. Applying Lemma 2.6 with $a=1, b=0, c=1, d=1$ and $r=1$, we obtain that $p_{z}(x+y) \leq p_{z}(x)+p_{z}(y)$ for $x, y \in \mathbf{C}^{n}$ and every $z \in \bar{G}$. Thus every $p_{z}, z \in \bar{G}$, is a norm.

(b) Since $p_{\zeta}(\cdot), \zeta \in \partial G$, are Hilbertian norms, $p_{\zeta}(x+y)^{2}+p_{\zeta}(x-y)^{2} \leq$ $2 p_{\zeta}(x)^{2}+2 p_{\zeta}(y)^{2}, x, y \in \mathbf{C}^{n}, \zeta \in \partial G$. Applying Lemma 2.6 with $a=1, b=$ $1, c=2, d=2, r=2$, we obtain that

$$
p_{z}(x+y)^{2}+p_{z}(x-y)^{2} \leq 2 p_{z}(x)^{2}+2 p_{z}(y)^{2}, \quad x, y \in \mathbf{C}^{n}, z \in \bar{G} .
$$

This implies that $p_{z}(\cdot)$ are Hilbertian norms (cf. Day [6, VII, §3]). (Actually Day mentions equations as the sufficient condition; note however that substituting in $(2.5)(x+y) / 2$ and $(x-y) / 2$ for $x$ and $y$ yields the opposite inequality, and so the equation.) Q.E.D.

The analog of Corollary 2.7(a) is, in general, false for $P_{Q}^{\text {psh }}$-interpolation methods studied in [18] (see Corollary 6.8 below for an example). For this reason, normed spaces were interpolated with help of classes $P_{Q}^{\text {co }}$, instead of $P_{Q}^{\text {psh }}$ (cf. [18, $\S \S 2$ and 8]). Recall that a function $u(z, w)$ belongs to $P_{\text {subh }}^{\text {co }}$ if it lies in $P_{\text {subh }}$ and the slice functions $w \rightarrow u(z, w)$ are locally convex for every $z$. As it was defined in [18], normed spaces $\left\{\mathbf{C}^{n},\|\cdot\|_{z}\right\}_{z \in \bar{G}}$ form a $P_{\text {subh }}^{\text {co }}$ interpolation family if the function $(z, w) \rightarrow\|w\|_{z}$ lies in $P_{\text {subh }}^{\mathrm{co}}$, and the set $X=\left\{(z, w) \in G \times \mathbf{C}^{n}:\|w\|_{z} \leq 1\right\}$ has the local maximum property with respect to class $P_{\text {subh }}^{\text {co }}$.

In view of Corollary $2.7(\mathrm{a})$, we have two, potentially different, methods, namely $P_{\text {subh }}$-interpolation and $P_{\text {subh }}^{\text {co }}$-interpolation, which both produce normed spaces out of normed boundary spaces. We will show now that they are consistent (in the normed case).

Proposition 2.8. Let $\left\{\boldsymbol{C}^{n},\|\cdot\|_{\zeta}\right\}_{\zeta \in \partial G}$ be a continuous family of normed spaces, where $G \subset \mathbf{C}^{k}$ is a bounded open set regular with respect to the Dirichlet problem. Let $\left\{\mathbf{C}^{n}, p_{z}(\cdot)\right\}_{z \in \bar{G}}$ be a continuous $P_{\text {subh }}$-interpolation family extending given boundary data (i.e. $p_{\zeta}=\|\cdot\|_{\zeta}, \zeta \in \partial G$ ). Then it is identical with the unique $P_{\text {subh }}^{\mathrm{co}}$-interpolation family of normed spaces extending given boundary data.

Proof. Denote by $\left\{\mathbf{C}^{n},\|\cdot\|_{z}\right\}_{z \in \bar{G}}$ the unique $P_{\text {subh }}^{\text {co }}$-interpolation family extending the given data (which exists by [18, Theorem 2.3]).

We denote $Y=\left\{(z, w) \in G \times \mathbf{C}^{n}: p_{z}(w) \leq 1\right\}$, and $X$ is defined as above. Then, $\bar{X} \backslash X=\bar{Y} \backslash Y=\left\{(\zeta, w) \in \partial G \times \mathbf{C}^{n}:\|w\|_{\zeta} \leq 1\right\}$. Let, further, $p(z, w)=$ $p_{z}(w)$ and $\varphi(z, w)=\|w\|_{z}$ for $(z, w) \in \bar{G} \times \mathbf{C}^{n}$. Then, $\varphi \mid G \times \mathbf{C}^{n} \in P_{\text {subh }}$, and so $\max \varphi|\bar{Y} \leq \max \varphi| \bar{Y} \backslash Y=\max \varphi \mid \bar{X} \backslash X=1$, which implies that

$$
Y \subset X \text {. }
$$

On the other hand, since the slice functions $p_{z}(\cdot)$ are convex by Corollary $2.7(\mathrm{a})$, therefore $p \in P_{\text {subh }}^{\text {co }}$, and since $X$ has the local maximum property relative to 
$P_{\text {subh }}^{\text {co }}$, we get $\max p|\bar{X} \leq \max p| \bar{X} \backslash X=\max p \mid \bar{Y} \backslash Y=1$, and so $X \subset Y$. In view of (2.6), $Y=X$, which implies identity of $p_{z}(w)$ and $\|w\|_{z}$. Q.E.D.

(Further information on the maximum principle employed in this proof can be found in [17, Corollary 4.4].)

We can now adapt the duality result of [18, Theorem 6.1 and $\S 8.2(\mathrm{~b})]$ to the context of this paper.

Theorem 2.9. If the normed spaces $\left\{\mathbf{C}^{n}, p_{z}(\cdot)\right\}_{z \in \bar{G}}$ form a harmonic interpolation family (i.e. $P_{\text {subh }}$-interpolation), then their dual normed spaces also form a harmonic interpolation family.

A new, direct proof of this duality theorem will be given in the sequel of this paper [21].

Remark. An analog of Corollary $2.7(\mathrm{a})$ is true with norms replaced by $r$-norms, i.e. quasinorms whose $r$ th power $(0<r \leq 1)$ satisfies the triangle inequality $p_{z}(x+y)^{r} \leq p_{z}(x)^{r}+p_{z}(y)^{r}, x, y \in \mathbf{C}^{n}$. If there is a common $r_{0} \in(0,1)$ such that this inequality holds for all $z \in \partial G$, then, applying Lemma 2.6 with $a=1, b=0, c=1, d=1$, and $r=r_{0}$, we obtain that it holds for all $z \in \bar{G}$, that is, all the $p_{z}$ 's are $r_{0}$-norms $(z \in \bar{G})$. The following, more precise, result will be proven in $\S 5$.

Theorem 2.10. In the situation of the last remark, denote by $r(z)$ the largest $r \in(0,1]$ such that the function $w \rightarrow p_{z}(w)^{r}: \mathbf{C}^{n} \rightarrow R$ is subadditive. Then the function $z \rightarrow 1 / r(z)$ is lsc and weak subharmonic (i.e. it satisfies the sub-meanvalue inequality).

The next corollary is related to the interpolation of uniformly convex and uniformly smooth spaces which will be studied in more detail in $\S 5$.

Corollary 2.11. Let $\left\{\mathbf{C}^{n}, p_{z}(\cdot)\right\}_{z \in \bar{G}}$ be a continuous harmonic interpolation family of quasinormed spaces. Then

(a) if there is $0<K<\infty$ such that the inequality $p_{z}(x+h)^{2}+p_{z}(x-h)^{2} \leq$ $2 p_{z}(x)^{2}+K p_{z}(h)^{2}$ for $x, h \in \mathbf{C}^{n}$, holds for all $z \in \partial G$, then this inequality holds for all $z \in \bar{G}$ (and $x, h \in \mathbf{C}^{n}$ ) with the same constant,

(b) if there is $c>0$ such that

$$
p_{\zeta}\left(x_{0}+h\right)^{2}+p_{\zeta}\left(x_{0}-h\right)^{2} \geq 2 p_{\zeta}\left(x_{0}\right)^{2}+c p_{\zeta}(h)^{2}
$$

for $x_{0}, h \in \mathbf{C}^{n}$ and $\zeta \in \partial G$, then this inequality holdls for all $\left(z, x_{0}, h\right) \in$ $\bar{G} \times \mathbf{C}^{n} \times \mathbf{C}^{n}$.

Proof. (a) Apply Lemma 2.6 with $a=1, b=1, c=2, d=K$ and $r=2$.

(b) By the substitution $x_{0}=\frac{1}{2}(x+y), h=\frac{1}{2}(x-y)$, the inequality (2.7) is equivalent to the inequality

$$
\frac{1}{2}\left(p_{\zeta}(x+y)\right)^{2}+\frac{c}{4}\left(p_{\zeta}(x-y)\right)^{2} \leq p_{\zeta}(x)^{2}+p_{\zeta}(y)^{2}, \quad(\zeta, x, y) \in G \times \mathbf{C}^{n} \times \mathbf{C}^{n} .
$$


The conclusion follows by applying Lemma 2.6 with $a=\frac{1}{2}, b=\frac{c}{4}, c=1, d=1$ and $r=2$. Q.E.D.

The following theorem will be used in [21] to study the regularity properties of the norm-function $(z, w) \rightarrow p_{z}(w)$.

Theorem 2.12. Let $\left\{\mathbf{C}^{n}, p_{z}\right\}_{z \in \bar{G}}$ be a (continuous) harmonic interpolation family of quasinormed spaces. Then

(a) if the functions $w \rightarrow p_{\zeta}(w)^{2}: \mathbf{C}^{n} \rightarrow R$ have $L^{\infty}$ second partial derivatives in $w$ and they are uniformly bounded (by a constant $C$ ) on $\partial G \times \mathbf{C}^{n}$, then the function $(z, w) \rightarrow p_{z}(w)^{2}$ has $L^{\infty}$ second partial derivatives in $w$ on $G \times \mathbf{C}^{n}$,

(b) if, in addition, the real Hessian in $w$ is positive definite for every $\zeta \in \partial G$ and a.a. $w \in \mathbf{C}^{n}$, and its lowest eigenvalue is greater or equal than a common constant $c>0$ (for $\zeta \in \partial G$, and a.e. $w \in \mathbf{C}^{n}$ ), then this property holds also for all $z \in G$ and a.e. $w \in \mathbf{C}^{n}$ (with the same constant).

Proof. (a) Let $C$ be an upper bound for the maximum eigenvalue of the real Hessian of $w \rightarrow p_{\zeta}(w)^{2}: \mathbf{C}^{n} \rightarrow R$ at a.e. $w \in \mathbf{C}^{n}$ and for all $\zeta \in \partial G$. By a standard criterion (cf. [12, Proposition 2.2]), the function $w \rightarrow p_{\zeta}(w)-\frac{1}{2} c|w|^{2}$ is concave for every $\zeta \in \partial G$, and so

$$
p_{\zeta}(x+y)^{2}+p_{\zeta}(x-y)^{2} \leq 2 p_{\zeta}(x)^{2}+C|h|^{2}, \quad x, h \in \mathbf{C}^{n},
$$

where $|\cdot|$ denotes the Euclidean norm on $\mathbf{C}^{n}$. Since $\left\{p_{\zeta}\right\}_{\zeta \in \partial G}$ is a continuous family of quasinorms, there is a positive constant $A$ such that

$$
A^{-1}|x| \leq p_{\zeta}(x) \leq A|x|, \quad \zeta \in \partial G, x \in \mathbf{C}^{n} .
$$

By this and (2.8), we get $p_{\zeta}(x+h)^{2}+p_{\zeta}(x-h)^{2} \leq 2 p_{\zeta}(x)^{2}+A^{2} C p_{\zeta}(h)^{2}$ for $\zeta \in \partial G, x, h \in \mathbf{C}^{n}$, and so, by Corollary $2.11(\mathrm{a})$,

$$
p_{z}(x+h)^{2}+p_{z}(x-h)^{2} \leq 2 p_{z}(x)^{2}+A^{2} C p_{z}(h)^{2} \text { for all } z \in G, x, h \in \mathbf{C}^{n} .
$$

Hence

$$
p_{z}(x+h)^{2}+p_{z}(x-h)^{2} \leq 2 p_{z}(x)^{2}+\left(A^{4} C\right)|h|^{2},
$$

because

$$
A^{-1}|x| \leq p_{z}(x) \leq A|x|, \quad z \in G, x \in \mathbf{C}^{n} .
$$

(The right-hand side of the last inequality follows from (2.9) and the subharmonicity of $z \rightarrow p_{z}(x)$; similarly, the left-hand side holds because the function $v(z)=\max \left\{x: p_{z}(x) \leq 1\right\}$ is subharmonic, since $z \rightarrow\left\{x: p_{z}(x) \leq 1\right\}$ is a harmonic multifunction.)

By (2.10), the function $x \rightarrow p_{z}(x)^{2}-\frac{1}{2} A^{4} C|x|^{2}$ is concave on $\mathbf{C}^{n}$ (for every $z \in G$ ), and so its distributional complex Hessian (in $x$ ) is a matrix-valued measure with values in the cone of negative semidefinite Hermitian matrices.

Denote the singular part of this matrix-valued measure by $\mu_{s}$. By the last remark, $\mu_{s}$ takes values in the cone of negative semidefinite Hermitian matrices; write informally $\mu_{s} \leq 0$. The matrix-valued measure representing the 
Hessian of $-\frac{1}{2} A C|x|^{2}$ is absolutely continuous with respect to the Lebesgue measure, and so $\mu_{s}$ is equal to the singular part of the Hessian measure of the plurisubharmonic function $(z, w) \rightarrow p_{z}(w)^{2}$. Consequently, $\mu_{s}$ takes values in the cone of positive semidefinite Hermitian matrices, shortly $\mu_{s} \geq 0$. Thus, $\mu_{s}=0$, and so both the complex Hessian and the real Hessian are absolutely continuous matrix valued measures. Since $w \rightarrow p_{z}(w)^{2}-\frac{1}{2} A^{4} C|w|^{2}$ is a concave function, the maximum eigenvalue of the real Hessian of $w \rightarrow p_{z}(w)^{2}$ is smaller than or equal to $\frac{1}{2} A^{4} C$ for a.a. $w$. In particular, second-order derivatives of $p_{z}(w)^{2}$ in $w$ are $L^{\infty}$ functions on $G \times \mathbf{C}^{n}$.

(b) The lower bound assumption for the Hessian implies that the function $x \rightarrow p_{\zeta}(x)^{2}-\frac{1}{2} c|x|^{2}$ is convex (for $\zeta \in \partial G$ ), which means that

$$
p_{\zeta}(x+h)^{2}+p_{\zeta}(x-h)^{2} \geq 2 p_{\zeta}(x)^{2}+c|h|^{2}, \quad x, h \in \mathbf{C}^{n}, \zeta \in \partial G,
$$

and so by (2.9),

$$
p_{\zeta}(x+h)^{2}+p_{\zeta}(x-h)^{2} \geq 2 p_{\zeta}(x)^{2}+\left(c / A^{2}\right) p_{\zeta}(h)^{2}, \quad x, h \in \mathbf{C}^{n}, \zeta \in \partial G .
$$

By Corollary $2.11(\mathrm{~b})$, this inequality persists in $G$

$$
p_{z}(x+h)^{2}+p_{z}(x-h)^{2} \geq 2 p_{z}(x)^{2}+\left(c / A^{2}\right) p_{z}(h)^{2}, \quad z \in G, x, h \in \mathbf{C}^{n},
$$

and by (2.11), we get $p_{z}(x+h)^{2}+p_{z}(x-h)^{2} \geq 2 p_{z}(x)^{2}+\left(c / A^{4}\right)|h|^{2}, z \in G$, $x, h \in \mathbf{C}^{n}$. Similarly as in the previous part, this inequality means that all the functions $x \rightarrow p_{z}(x)^{2}-\left(c / 2 A^{4}\right)|x|^{2}, z \in G$, are convex on $\mathbf{C}^{n}$. Hence, the real Hessian matrix in $x$ (whose components are already known to be $L^{\infty}$ functions) has a.e. lowest eigenvalue greater than or equal to $c / A^{4}$. Q.E.D.

\section{FURTHER PROPERTIES OF HARMONIC MULTIFUNCTIONS. EXAMPLES}

In this section we prove additional results on harmonic multifunctions which will be needed in the following sections. As a by-product, we obtain the next theorem.

Theorem 3.1. Let $q(z)>0, z \in G, G \subset \mathbf{C}^{k}$, where $G$ is open. Then the quasinormed spaces $\left\{l_{q(z)}^{m}\right\}_{z \in G}$ form a harmonic interpolation family (resp. sub-or superinterpolation) if and only if the function $z \rightarrow 1 / q(z): G \rightarrow R_{+}$is harmonic (resp. sub- or superharmonic).

In the normed case, i.e. when $q(z)>1$ for $z \in G$, this result was first proved by Coifman and Semmes (by an apparently different method) and announced in [5] (see also Rochberg [10]).

Proposition 3.2. If $z \rightarrow K(z): G \rightarrow 2^{\mathrm{C}^{n}}, G \subset \mathbf{C}^{k}$, is a harmonic multifunction and $(z, w) \rightarrow F_{z}(w): G \times \mathbf{C}^{n} \rightarrow \mathbf{C}^{m}$ is a holomorphic mapping, then $z \rightarrow$ $F_{z}(K(z)): G \rightarrow 2^{\tilde{\mathrm{C}}^{m}}$ is a harmonic multifunction.

Proof. Denote $L(z)=F_{z}(K(z))$. Let $u(z, x, \xi)$ be a $P_{\text {subh }}$ function on (a subset of) $G \times \mathbf{C}^{m+s}$, and let $v(z, \xi)=\max \{u(z, x, \xi): x \in L(z)\}$. Since $v(z, \xi)=$ 
$\max \left\{u\left(z, F_{z}(w), \xi\right): w \in K(z)\right\}$ and the function $(z, w, \xi) \rightarrow u\left(z, F_{z}(w), \xi\right)$ is $P_{\text {subh }}$ on a subset of $G \times \mathbf{C}^{n+s}$ (by property (1.2)), $v \in P_{\text {subh }}$. Q.E.D.

Proposition 3.3. Let $z \rightarrow K_{t}(z): G \rightarrow 2^{\mathrm{C}^{n}}, t \in T$, (where $T$ is an index set) be harmonic multifunctions. Assume that the multifunction $K(z):=\bigcup_{t \in T} K_{t}(z)$ is locally uniformly bounded in $G$. Then its usc regularization, $K^{*}(z)=$ Lim Sup $\operatorname{Su}_{x \rightarrow z} K(x)$ is a harmonic multifunction. In particular, $z \rightarrow K(z): G \rightarrow$ $2^{\mathrm{C}^{n}}$ is a harmonic multifunction, provided it is usc.

Proof (sketch). The claim follows immediately from Definition 2.1 and from the property of the class $P_{\text {subh }}$ that, if $v_{t}(z, \xi)_{t \in T}$ is a family of $P_{\text {subh }}$-functions which is locally uniformly upper bounded, then the usc regularization of their supremum belongs to $P_{\text {subh }}$. (The latter property of $P_{\text {subh }}$ is a direct consequence of the definition of $P_{\text {subh }}$ and the analogous property of "subh".) Q.E.D.

The next fact is a special case of [18, Theorem 6.9]. We sketch here a simpler proof.

Proposition 3.4. If $\left\{\mathbf{C}^{n}, p_{z}(\cdot)\right\}_{z \in G}$ is a harmonic subinterpolation family of quasinormed spaces and $h: G \rightarrow R$ is a subharmonic function, then the quasinormed spaces $\left\{\mathbf{C}^{n}, e^{h(z)} p_{z}(\cdot)\right\}_{z \in G}$ form also a harmonic subinterpolation family.

If spaces $\left\{\mathbf{C}^{n}, p_{z}(\cdot)\right\}_{z \in G}$ form a harmonic interpolation family (resp. superinterpolation) and $h: G \rightarrow R$ is a harmonic (resp. superharmonic) function, then $\left\{\mathbf{C}^{n}, e^{h(z)} p_{z}(\cdot)\right\}_{z \in G}$ is an interpolation (resp. superinterpolation) family.

Proof. The first part follows directly from Remark 2.5(b). By Remark 2.5(a), the statement for harmonic interpolation is an obvious consequence of those for sub- and superinterpolation. It remains to consider the latter case.

Let $K(z)=\left\{w: p_{z}(w) \leq 1\right\}$. Then the set $e^{-h(z)} K(z)$ is the unit ball of the quasinorm $e^{h(z)} p_{z}(\cdot), z \in G$.

Similarly as in $[18, \S \S 2,3]$, we will call a family $\{W(z)\}_{z \in G}, W(z) \subset C^{n}$, of quasinorming bodies (=compact strictly star-shaped neighborhoods of zero in $\mathbf{C}^{n}$ ) a harmonic interpolation family if the copies of $\mathbf{C}^{n}$ equipped with corresponding quasinorms (i.e. Minkowski's functionals of $W(z)$ ) form a harmonic interpolation family. Analogous terminology will be used for sub- and superinterpolation families.

We have to show that $\left\{e^{-h(z)} K(z)\right\}_{z \in G}$ is a (harmonic) superinterpolation family. For this, consider a Euclidean ball $\bar{B}_{0} \subset G$ and a subinterpolation family $\{W(z)\}_{z \in \bar{B}_{0}}$, such that $e^{-h(\zeta)} K(\zeta) \subset W(\zeta), \zeta \in \bar{B}_{0}$. By the definition following Proposition 2.4, it suffices to check that $e^{-h(z)} K(z) \subset W(z), z \in B_{0}$.

By the first part of the statement, $\left\{e^{h(z)} W(z)\right\}_{z \in \bar{B}_{0}}$ is a subinterpolation family. Since $e^{h(\zeta)} W(\zeta) \supset K(\zeta), \zeta \in \partial B_{0}$, we get $e^{h(z)} W(z) \supset K(z), z \in$ $B_{0} \quad\left(\{K(\cdot)\}\right.$ is a superinterpolation family), and so $W(z) \supset e^{-h(z)} K(z), z \in$ $B_{0}$. Q.E.D. 
Lemma 3.5. Let $h: G_{0} \rightarrow R$ be a superharmonic function, $m$ be a positive integer, and

$$
K(z)=\left\{w \in \mathbf{C}^{m}: \sum_{i=1}^{m}\left|w_{i}\right|^{1 / h(z)} \leq 1\right\}, \quad z \in G_{0} .
$$

Then $z \rightarrow K(z): G_{0} \rightarrow 2^{\mathrm{C}^{m}}$ is a harmonic multifunction. In other words, the quasinormed spaces $\left\{l_{1 / k(z)}^{m}\right\}_{z \in G_{0}}$ form a harmoic superinterpolation family.

Proof. We prove first the following assertion.

Assertion. If $r \in(0,1)$ and $h: G_{0} \rightarrow R$ is superharmonic, then the multifunction $L(z)=\left\{\alpha \in \mathbf{C}:|\alpha| \leq r^{h(z)}\right\}$ is harmonic.

This is a special case of [18, Assertion 1 in the proof of Lemma 2.8]. I can also be obtained from Proposition 3.4 as follows. Using Definition 2.1, one checks easily that the constant family of norming bodies, $L_{0}(z)=\{\alpha \in \mathbf{C}:|\alpha| \leq 1\}$, is a harmonic multifunction, and so, by Proposition 2.4, a harmonic superinterpolation family. Since $L(z)=e^{(\log r) h(z)} L_{0}(z), z \in G$, and $(\log r) h(z)$ is subharmonic, $z \rightarrow L(z)$ is a harmonic superinterpolation family by Proposition 3.4, and so a harmonic multifunction (cf. Proposition 2.4). (A direct proof analogous to that of Assertion 1 in the Proof of Theorem 4.1 below, can also be given.)

By the assertion and Proposition 2.3, every multifunction of the form

$$
z \rightarrow\left\{\left(\zeta_{1}, \ldots, \zeta_{m}\right):\left|\zeta_{i}\right| \leq r_{i}^{h(z)}, i=1, \ldots, m\right\}: G_{0} \rightarrow 2^{\mathrm{C}^{n}}
$$

is harmonic, provided $0<r_{i}<1, i=1, \ldots, m$. Since $K(z), z \in G_{0}$, is the union of the sets (3.2), with $r_{1}+\cdots+r_{m} \leq 1$, and of the sets

$$
\left\{\left(\zeta_{1}, \ldots, \zeta_{m}\right) \in \mathbf{C}^{m}: \zeta_{1}=\cdots=\zeta_{i-1}=\zeta_{i+1}=\cdots=\zeta_{m}=0,\left|\zeta_{i}\right| \leq 1\right\},
$$

$i=1, \ldots, m$, then $z \rightarrow K(z): G_{0} \rightarrow 2^{\mathrm{C}^{m}}$ is a harmonic multifunction by Proposition 3.3.

Since $K(z)$ are unit balls of the quasinormed spaces $\left\{l_{1 / h(z)}^{m}\right\}_{z \in G_{0}}$, the latter form a harmonic subinterpolation family by Proposition 2.4. Q.E.D.

Proof of Theorem 3.1. Sufficiency. The case of superinterpolation families was covered in the last lemma.

Assertion. If $q(z)>1, z \in G_{0}$, and $1 / q(z)$ is a subharmonic function, then $\left\{l_{q(z)}^{m}\right\}_{z \in G_{0}}$ form a harmonic subinterpolation family of normed spaces.

To prove it, denote by $r(z)$ the conjugate exponent, i.e. $q(z)^{-1}+r(z)^{-1}=1$. Then $r(z)>1$ in $G_{0}$ and $1 / r(z)=1-1 / q(z)$ is a superharmonic function. Hence, the spaces $\left\{l_{r(z)}^{m}\right\}_{z \in G_{0}}$ form a harmonic superinterpolation family, and so, by Theorem 2.9, or a simple duality result [18, Proposition 4.3], their dual normed spaces, $\left\{l_{q(z)}^{m}\right\}_{z \in G_{0}}$, form a harmonic subinterpolation family, which proves the assertion. We will now reduce the quasinormed case $(q(z)<1)$ to the normed case. 
Now fix a positive integer $k$, and let $G_{k}=\{z \in G: q(z)>1 / k\}$. Denote $p(z, w)=\|w\|_{q(z)}, z \in G, w \in \mathbf{C}^{n}$, and $\tilde{p}(z, v)=\|v\|_{k q(z)}, z \in G_{k}, v \in \mathbf{C}^{n}$. Since $k q(z)>1$ on $G_{k}$, the function $\log \tilde{p}(z, w)$ is of class $P_{\text {subh }}$ on $G_{k} \times \mathbf{C}^{m}$, by the assertion and Remark $2.5(\mathrm{~b})$. Observe that

$$
\log p\left(z ; w_{1}, \ldots, w_{m}\right)=k \log \tilde{p}\left(z ; w_{1}^{1 / k}, \ldots, w_{m}^{1 / k}\right)
$$

(where the roots can be chosen in an arbitrary fashion). By applying property (1.2) locally (to holomorphic branches of the roots) in $G_{k} \times\left(\mathbf{C}^{m} \backslash\{0\}\right)$, one concludes that $\log p \mid G_{k} \times\left(\mathbf{C}^{m} \backslash\{0\}\right)$ is $P_{\text {subh }}$; since $\log p=-\infty$ on $G_{k} \times\{0\}$, it is $P_{\text {subh }}$ on $G_{k} \times \mathbf{C}^{n}$ also.

Since $\bigcup_{k=1} G_{k}=G, \log p$ belongs to $P_{\text {subh }}\left(G \times \mathbf{C}^{n}\right)$ (the class $P_{\text {subh }}$ is local), and so the spaces $\left\{l_{q(z)}^{m}\right\}_{z \in G}$ form a harmonic subinterpolation family by Remark 2.5(b).

If $1 / q(z)$ is a harmonic function, $\left\{l_{q(z)}^{m}\right\}_{z \in G}$ form both a super- and subinterpolation family, and so an interpolation family, by Lemma 2.5(a).

Necessity. Necessity is obvious in the interpolation case, by the reiteration property [18, Remark 2.10] and the "sufficiency" part. The latter, together with [18, Definition 3.3], yields the characterization of sub- and superinterpolation families. We omit further details. Q.E.D.

\section{CONSTRUCTING NEW INTERPOLATION FAMILIES BY COMPOSITION AND DIRECT SUM}

We say that a one-parameter family of normed spaces $\left\{\mathbf{C}^{n}, N_{t}(\cdot)\right\}_{0<t<1}$ is a Calderón interpolation family if for every $\alpha, \beta \in(0,1), \alpha<\beta$, the normed spaces $\left\{\mathbf{C}^{n}, N_{t}\right\}_{\alpha \leq t \leq \beta}$ are obtained by the complex interpolation method of Calderón from the boundary spaces $\left(\mathbf{C}^{n}, N_{\alpha}(\cdot)\right)$ and $\left(\mathbf{C}^{n}, N_{\beta}(\cdot)\right)$. In view of the results of Coifman et al. [2,4] and [18, $\S 7.1(\mathrm{a})]$, one can conclude easily that $\left\{\mathbf{C}^{n}, N_{t}(\cdot)\right\}_{0<t<1}$ is a Calderón interpolation family if and only if the family $\left\{\mathbf{C}^{n}, N_{\operatorname{Re} z}(\cdot)\right\}$ is a harmonic interpolation family on the strip $\{z \in \mathbf{C}: 0<$ $\operatorname{Re} z<1\}$.

By analogy, we say that a family $\left\{\mathbf{C}^{n}, N_{t}\right\}_{0<t<t_{0}}$ (where $t_{0} \leq+\infty$ ) is a Calderón interpolation family of quasinormed spaces if the family $\left\{\mathbf{C}^{n}, N_{\operatorname{Re} z}(\cdot)\right\}$ is a harmonic interpolation family of quasinormed spaces in the domain $\{z \in$ C: $\left.0<\operatorname{Re} z<t_{0}\right\}$.

We can formulate now the main result of this section. It answers a question posed by Rochberg [10, $\S 3 \mathrm{C}]$.

Theorem 4.1. Let $\left\{\mathbf{C}^{n}, N_{t}(\cdot)\right\}_{t \in\left(0, t_{0}\right)}$, where $0<t_{0} \leq \infty$, be a Calderón interpolation family of quasinormed spaces. Let $h: G \rightarrow\left(0, t_{0}\right)$ (where $G$ is an open set in $\left.\mathbf{C}^{k}\right)$. Then the spaces $\left\{\mathbf{C}^{n}, N_{h(z)}(\cdot)\right\}_{z \in G}$ form a harmonic interpolation family.

To establish this composition theorem, we consider separately the subinterpolation property and the superinterpolation property. 
Remark 4.2. Recall that, in the notation of [19, Remark 4.8], $P_{\mathrm{psh}}^{\mathrm{psh}}=P_{0}$, where $P_{0}=$ psh stands for the class of all plurisubharmonic functions on $\mathbf{C}^{k} \times \mathbf{C}^{n}$. By [18, Theorem 4.1], quasinormed spaces $\left\{\mathbf{C}^{n}, p_{x}(\cdot)\right\}_{x \in H}, H \subset \mathbf{C}^{n}$, form a $P_{0}$-subinterpolation family if and only if the function $(x, w) \rightarrow p_{x}(w)$ is plurisubharmonic on $H \times \mathbf{C}^{m}$. If $H$ is a simply-connected planar domain, this notion is identical with that of a subinterpolation family in the sense of Rochberg [9, §2]. (Our main application will be in the case of a planar domain $H$.)

Corollary 4.3. Let $z \rightarrow K(z): G \rightarrow 2^{H}$, where $G \rightarrow \mathbf{C}^{k}, H \subset \mathbf{C}^{n}$, be a harmonic multifunction, and let $\left\{\mathbf{C}^{m}, p_{x}(\cdot)\right\}_{x \in H}$ be a $P_{0}$-subinterpolation family (of quasinormed spaces). Define $q_{z}(w)=\max \left\{p_{x}(w): x \in K(z)\right\}$. Then the quasinormed spaces $\left\{\mathbf{C}^{n}, q_{z}(\cdot)\right\}_{z \in G}$ form a harmonic subinterpolation family.

Proof. By Remark 4.2, the function $u(z, x, w)=p_{x}(w)$ is plurisubharmonic; in particular $u \in P_{\text {subh }}\left(G \times \mathbf{C}^{n+m}\right)$. Since $K(\cdot)$ is a harmonic multifunction and $q_{z}(w)=\max \{u(z, x, w): x \in K(z)\}$, the function $(z, w) \rightarrow q_{z}(w)$ belongs to $P_{\text {subh }}\left(G \times \mathbf{C}^{m}\right)$, which implies the conclusion by Remark 2.5(b). Q.E.D.

Recall that a usc, compact-valued multifunction $z \rightarrow K(z): H \rightarrow 2^{\mathrm{C}^{m}}, H \subset$ $\mathbf{C}^{n}$, is called analytic if the intersection of its graph, $\operatorname{gr}(K)=\{(z, w): z \in$ $H, w \in K(z)\}$, with every $(m+1)$-dimensional complex plane in $\mathbf{C}^{n+m}$ has local maximum property with respect to plurisubharmonic functions (cf. [15, Definition 0.2; 13]).

Remark 4.4. When $H$ is a planar domain $(n=1)$, the notions of harmonic and analytic multifunctions are identical. (In this case $P_{\text {subh }}=P_{0}=\mathrm{psh}$; cf. property (1.5).)

Theorem 4.5. Let $z \rightarrow L(z): G \rightarrow 2^{P}$ (where $G \subset \mathbf{C}^{k}$ and $P \subset \mathbf{C}^{n}$ are open sets) be a harmonic multifunction, and let $x \rightarrow K(x): P \rightarrow 2^{\mathrm{C}^{m}}$ be an analytic multifunction. Then their composition $(K \circ L)(z):=\bigcup_{x \in L(z)} K(x)$ is a harmonic multifunction, $z \rightarrow(K \circ L)(z): G \rightarrow 2^{\mathrm{C}^{m}}$.

The result is no longer true, if one assumes that $L(\cdot)$ is analytic and $K(\cdot)$ is harmonic; similarly, the order of composition cannot be reversed in Proposition 3.2 .

Note that this is consistent with the failure of a similar property in the case of harmonic functions: if $G \subset \mathbf{C}^{k}, P \subset \mathbf{C}^{n}, F: G \rightarrow P$ is analytic, $u: P \rightarrow R$ is harmonic (or subharmonic), and $n>1$, then $u \circ F$ is usually not harmonic (subharmonic).

The affirmative property that holds for multifunctions does not have an automatic analog for functions, but the following may serve as a close approximation. If $h: G \rightarrow R$ is a harmonic function, $G \subset \mathbf{C}^{k}$, and $F: G \times \mathbf{C}^{n} \rightarrow \mathbf{C}$ is an analytic function, then the function $v(z)=\max \left\{|F(z, w)|:|w| \leq e^{h(z)}\right\}$ is a subharmonic function. 
Proof of Theorem 4.5. Denote $R(z)=K \circ L(z), z \in G$. Consider an open set $H \subset G \times \mathbf{C}^{m+n}$ and a function $u(z, w, \xi)$ of class $P_{\text {subh }}(H)$ (relative to the factorization $\left.G \times \mathbf{C}^{m+s}\right)$. According to Definition 2.1, we have to show that the function $v(z, \xi)=\max \{u(z, w, \xi): w \in R(z)\}$ is of class $P_{\text {subh }}\left(H_{1}\right)$, where $H_{1}=\left\{(z, \xi) \in G \times \mathbf{C}^{S}:\{z\} \times R(z) \times\{\xi\} \subset H\right\}$. Let $\varphi(z, y, \xi)=$ $\max \{u(z, w, \xi): w \in K(y)\}$. Clearly, $v(z, \xi)=\max \{\varphi(z, x, \xi): x \in L(z)\}$ for $(z, \xi) \in H_{1}$. Seeing that $z \rightarrow L(z): G \rightarrow 2^{\mathrm{C}^{n}}$ is a harmonic multifunction, it suffices now to show that the function $\varphi(z, x, \xi)$ is of class $P_{\text {subh }}$ on the set $\left\{(z, x, \xi) \in G \times \mathbf{C}^{n+s}:\{z\} \times K(x) \times\{\xi\} \subset H\right\}$. To check this consider an arbitrary holomorphic map $z \rightarrow(f(z), g(z)): G_{0} \rightarrow \mathbf{C}^{m} \times \mathbf{C}^{n}, G_{0} \subset G$; it is required that the function $\chi(z)=\varphi(z, f(z), g(z))$ is subharmonic in $G_{0}$. Clearly,

$$
\chi(z)=\max \{u(z, w, g(z)): w \in K(f(z))\}, \quad z \in G_{0} .
$$

Since the function $(z, w) \rightarrow u(z, w, g(z))$ is of class $P_{\text {subh }}$ on a subset of $G \times \mathbf{C}^{n}$ (by property (1.2)), (4.1) and the following assertion imply that $\chi(\cdot)$ is a subharmonic function.

Assertion 1. The multifunction $z \rightarrow K(f(z)): G_{0} \rightarrow 2^{\mathrm{C}^{m}}$ is harmonic.

The proof of Assertion 1 relies on the following fact.

Assertion 2. If $z \rightarrow K(z): P \rightarrow 2^{\mathbf{C}^{m}}, P \subset \mathbf{C}^{n}$, is an analytic multifunction and $f: G_{0} \rightarrow P, G_{0} \subset \mathbf{C}^{k}$, is an analytic mapping, then $z \rightarrow K(f(z)): G_{0} \rightarrow 2^{\mathbf{C}^{m}}$ is an analytic multifunction.

By this and [14, Theorem 5.1], the set $Y=\operatorname{gr}(K \circ f)=\left\{(z, w) \in G_{0} \times\right.$ $\left.\mathbf{C}^{\prime n}: w \in K(f(z))\right\}$ has the local maximum property with respect to $(k-1)$ plurisubharmonic functions; since $P_{\text {subh }} \subset P_{k-1}$ (cf. (1.4)), $Y$ has the local maximum property with respect to the class $P_{\text {subh }}$, and so $z \rightarrow K(f(z))$ is a harmonic multifunction by Proposition 2.2.

It remains to prove Assertion 2. Denote $X=\operatorname{gr}(K)=\{(x, w): x \in P, w \in$ $K(z)\}$. Then $X$ is an (n-1)-maximum set (cf. [14, Definition 2.1]), and $G \times X$ is a $(k+n-1)$-maximum set in $G \times P \times \mathbf{C}^{m}$ by [14, Assertion 1 in the proof of Corollary 2.8]. Consider now the set

$$
(G \times X) \cap\left\{(z, x, w) \in G \times P \times \mathbf{C}^{m}: f(z)=x\right\} .
$$

Since the intersection (4.2) is defined by $n$ equations, $f_{i}(z)=x_{i}, i=1, \ldots, n$, the set $(4.2)$ is a $(k-1)$-maximum set by [14, Corollary 2.6] or [14, Theorem 2.7].

Observe now that the restriction of the holomorphic map $(z, x, w) \rightarrow(z, w)$ to the set (4.2) maps the latter in a one-to-one fashion onto $Y=\operatorname{gr}(K \circ f)$, and so $Y$ is a $(k-1)$-maximum set (cf. e.g. [14, Lemma 3.6]). Thus, $K \circ f$ is an analytic multifunction, which proves Assertion 2. Q.E.D.

Proof of Theorem 4.1. Let $L_{1}(z)=\left\{x \in \mathbf{C}:|x|=e^{h(z)}\right\}, z \in G$, and $P=$ $\left\{x \in \mathbf{C}: 1<|x|<e^{t_{0}}\right\}$. Denote $p_{x}(w)=N_{\log |x|}(w)$ for $x \in P, w \in \mathbf{C}^{n}$, and $K(x)=\left\{w \in \mathbf{C}^{n}: p_{x}(w) \leq 1\right\}, x \in P$. 
Assertion 1. The multifunction $z \rightarrow L_{1}(z): G \rightarrow 2^{\mathrm{C}}$ is harmonic.

Assertion 2. Quasinormed spaces $\left\{\mathbf{C}^{m}, p_{x}(\cdot)\right\}_{x \in P}$ form a $P_{0}$-subinterpolation family and $x \rightarrow K(x): P \rightarrow 2^{\mathrm{C}^{n}}$ is an analytic multifunction.

These assertions allow for the reduction of Theorem 4.1 to already established composition results. We show this first.

Denote $q_{z}(w)=N_{h(z)}(w), z \in G, w \in \mathbf{C}^{n}$, and $W(z)=\left\{w \in \mathbf{C}^{n}: q_{z}(w) \leq\right.$ $1\}$. Since both $p_{x}(w)$ and $K(x)$ depend only on the value of $\log |x|$, we have

$$
\begin{aligned}
& W(z)=\left(K \circ L_{1}\right)(z), \quad z \in G, \\
& q_{z}(w)=\max \left\{p_{x}(w): x \in L_{1}(z)\right\}, \quad z \in G .
\end{aligned}
$$

Since $L_{1}$ is a harmonic multifunction, the first relation, Assertion 2 and Theorem 4.5 imply that the multifunction $z \rightarrow W(z): G \rightarrow 2^{\mathrm{C}^{n}}$ is harmonic. Since $W(z), z \in G$, are unit balls of the quasinorms $q_{z}(\cdot)$, we conclude from Proposition 2.4 that the spaces $\left\{\mathbf{C}^{n}, q_{z}(\cdot)\right\}_{z \in G}=\left\{\mathbf{C}^{n}, N_{h(z)}(\cdot)\right\}_{z \in G}$ from a (harmonic) superinterpolation family. These spaces form also a subinterpolation family by Proposition 4.3, Assertion 2 and relation (4.3). Consequently, $\left\{\mathbf{C}^{n}, N_{h(z)}\right\}_{z \in G}$ is a harmonic interpolation family, as required. It remains to prove the assertions.

Proof of Assertion 1. Choose a $C^{(2)}$-smooth function $\varphi: R \rightarrow R$ such that $\varphi(0)=0$ and $\varphi(t)<0$ for $t=0$. Let $u(z, w)=\varphi(h(z)-\log |w|), \quad z \in G$, $w \neq 0$. We show first that $u \in\left(P_{\text {subh }}\right)^{d}=$ the dual class to $P_{\text {subh }}$ (cf. Terminology 1.1). Fix $(a, b) \in G \times(\mathbf{C} \backslash\{0\})$. According to the criterion of Remark 1.2, it suffices to show that there is a C-linear map $S: \mathbf{C}^{k} \rightarrow \mathbf{C}$, such that the form

$$
z \rightarrow \operatorname{Hess}^{\mathbf{C}} u_{(a, b)}(z \oplus S z, z \oplus S z)
$$

has nonnegative trace. Seeing that

$$
\operatorname{Hess}^{\mathrm{c}} u_{(a, b)}=\varphi^{\prime}(r)\left(\operatorname{Hess}_{a}^{\mathrm{c}} h+0\right)+\varphi^{\prime \prime}(r) \bar{D}_{a, b} \otimes D_{a, b},
$$

where $r=h(a)-\log |b|$, and $D_{a, b}$ stands for the first-order differential at $(a, b)$ of the function $h(z)-\log |w|$, we choose $S: \mathbf{C}^{k} \rightarrow \mathbf{C}$, so that the graph of $S$ is equal to the kernel of $D_{a, b}: \mathbf{C}^{k+1} \rightarrow \mathbf{C}$. Then the form (4.4) is equal to $\varphi^{\prime}(r) \operatorname{Hess}_{a}^{\mathrm{C}} h(z, z)$, and has zero trace. Thus

$$
u \in\left(P_{\text {subh }}\right)^{d}(G \times(\mathbf{C} \backslash\{0\})) .
$$

Observe that $\operatorname{gr}\left(L_{1}\right)=\{(z, w) \in G \times(\mathbf{C} \backslash\{0\}): u(z, w)=0\}$, and $u<0$ outside $\operatorname{gr}\left(L_{1}\right)$. Hence $n u(z, w) \searrow \chi(z, w), z \in G, w \neq 0$, where $\chi \mid \operatorname{gr}\left(L_{1}\right)=$ 0 , and $\chi \mid(G \times(\mathbf{C} \backslash\{0\})) \backslash \operatorname{gr}\left(L_{1}\right)=-\infty$. One can show easily that the dual class of functions contains the limit of a decreasing sequence of functions from this class, and so $\chi \in P_{\text {subh }}^{d}$ by (4.5). It remains to show (by Proposition 2.2) that the set $X=\operatorname{gr}\left(L_{1}\right)$ has the local maximum property relative to $P_{\text {subh }}$. Let $K \subset G \times(\mathbf{C} \backslash\{0\})$ be compact and such that $K \cap X$ is compact and $u \in P_{\text {subh }}$ 
near $K$. By the local maximum property of $u+\chi$ (on open subsets of $\mathbf{C}^{n+1}$ ) and the fact that $\chi \equiv-\infty$ off $X$, we get $\max u|K \cap X=\max (u+\chi)| K \leq$ $\max (u+\chi)|\partial K=\max u| X \cap(\partial K)$. Thus, $L_{1}$ is a harmonic multifunction. (More general versions of these arguments can be found in [17, Proposition 4.6 and Lemma 2.9(i)].) Q.E.D.

Proof of Assertion 2. The definition of a Calderón interpolation family, formulated before Theorem 4.1, amounts to the requirement that the function

$$
(\xi, w) \rightarrow N_{\operatorname{Re} \xi}(w): H \times \mathbf{C}^{n} \rightarrow R
$$

be $P_{\text {subh }}$ on $H \times \mathbf{C}^{n}$ and that the multifunction $\xi \rightarrow K\left(e^{\xi}\right): H \rightarrow 2^{\mathrm{C}^{n}}$ be harmonic. Since $H$ is planar, and $P_{\text {subh }}=P_{0}(=\mathrm{psh})$ on $H \times \mathbf{C}^{n}$ by $(1.5)$, the function (4.6) is plurisubharmonic. Substituting locally a branch of $\xi=\log x$, one obtains that the function $(x, w) \rightarrow p_{x}(w): P \times \mathbf{C}^{n} \rightarrow R$ is plurisubharmonic, i.e. spaces $\left\{\mathbf{C}^{n}, p_{x}(\cdot)\right\}_{x \in P}$ form a $P_{0}$-subinterpolation family.

By Remark 4.4, $\xi \rightarrow K\left(e^{\xi}\right)$ is an analytic multifunction in $H$. Using locally the substitution $\xi=$ (a branch of) $\log x$ (cf. e.g. Assertion 2 in the proof of Theorem 4.5 above, or the simpler fact [11, Proposition 5.1], or use a direct argument), we obtain that $x \rightarrow K(x): P \rightarrow 2^{\mathrm{C}^{n}}$ is locally an analytic multifunction. Since the notion of an analytic multifunction is local (cf. [15, Definition 0.2]), this concludes the proof of Assertion 2. Q.E.D.

Remark. Theorem 3.1 can now also be obtained by application of the composition method of Theorem 4.1 and the fact that the spaces $\left\{l_{1 / t}^{n}\right\}_{0<t<\infty}$ form a Calderón interpolation family (in the sense of Theorem 4.1).

We show now that harmonic interpolation families behave well under the operation of direct sum.

Proposition 4.6. Let $0<r \leq+\infty$. Assume that $\left\{\mathbf{C}^{n}, p_{z}(\cdot)\right\}_{z \in G},\left\{\mathbf{C}^{m}, q_{z}(\cdot)\right\}_{z \in G}$ (where $G \subset \mathbf{C}^{k}$ ) are harmonic interpolation (resp. sub-or superinterpolation) families of quasinormed spaces. Then the spaces $\left\{\mathbf{C}^{n+m}, N_{z}^{r}(\cdot)\right\}_{z \in G}$ form a harmonic interpolation (resp. sub-or superinterpolation) family, where $N_{z}^{r}(x \oplus y)=$ $\left(p_{z}(x)^{r}+q_{z}(y)^{r}\right)^{1 / r}$ if $0<r<+\infty$, and $N_{z}^{\infty}(x \oplus y)=\max \left(p_{z}(x), q_{z}(y)\right)$.

Proof. Once again, it suffices to consider only the cases of sub- and superinterpolation families.

Case 1. Subinterpolation. Assume first that $0<r<\infty$. Since the functions $(z, x) \rightarrow \log p_{z}(x)$ and $(z, y) \rightarrow \log p_{z}(y)$ are $P_{\text {subh }}$, then the functions $p_{z}(x)^{r}$ and $p_{z}(y)^{r}$ are also of the class $P_{\text {subh }}$, by (1.7), on $G \times \mathbf{C}^{n}$ and $G \times \mathbf{C}^{m}$ respectively. By properties (1.2) and (1.1), the function $u(z, x, y)=p_{z}(x)^{r}+$ $p_{z}(y)^{r}$ belongs to $P_{\text {subh }}\left(G \times \mathbf{C}^{n+m}\right)$. Since $u$ satisfies the homogeneity condition $u(z, \lambda x, \lambda y)=|\lambda|^{r} u(z, x, y), z \in G, x \in \mathbf{C}^{n}, y \in \mathbf{C}^{m}$, the function log $u$ must belong to $P_{\text {subh }}$. (This was shown in [18, Proof of Theorem 4.1, claim (4.1) and following comments] for the case $r=1$, but the proof for the general case $r>1$ is practically identical.) Hence, $u^{1 / r} \in P_{\text {subh }}$ by property (1.7). 
The case $r=\infty$ is obvious, because the class $P_{\text {subh }}$ is closed with respect to the operation of taking the maximum of several functions (which follows directly from the definition of the class $P_{\text {subh }}$ ).

Case 2. Superinterpolation. Let $K(z), L(z)$ and $R(z)$ denote the unit balls of the quasinorms $p_{z}(\cdot), q_{z}(\cdot)$ and $N_{z}^{r}(\cdot)$ respectively. We know that the multifunctions $z \rightarrow K(z): G \rightarrow 2^{\mathrm{C}^{n}}$ and $z \rightarrow L(z): G \rightarrow 2^{\mathrm{C}^{m}}$ are harmonic, and it remains to show that $z \rightarrow R(z): G \rightarrow 2^{\mathrm{C}^{n+m}}$ is a harmonic multifunction as well (cf. Proposition 2.4). In case $r=\infty, R(z)=K(z) \times L(z), z \in G$, and the conclusion follows by Proposition 2.3. In case $0<r<\infty$,

$$
R(z)=\bigcup\left\{t K(z) \times s L(z): t, s \geq 0, t^{r}+s^{r}=1\right\} .
$$

Since for each pair $t, s$ the multifunction

$$
z \rightarrow t K(z) \times s L(z): G \rightarrow 2^{\mathrm{C}^{n+m}}
$$

(where $t K(z)=\{t x: x \in K(z)\}$ ) is harmonic by Propositions 2.3 and 3.2, Proposition 3.3 and (4.7) imply that $R(\cdot)$ is a harmonic multifunction. Q.E.D.

\section{INTERPOLATIONS OF UNIFORMLY CONVEX AND UNIFORMLY SMOOTH SPACES}

In this section we generalize Corollary 2.11 to arbitrary strictly convex and smooth spaces. In case the modulus of convexity or of smoothness is of power type, the reciprocal of the power type exponent is shown to be a super- or subharmonic function, similarly to the situation in Theorem 2.10 (which will also be proven in this section).

For general background on moduli of convexity or smoothness of normed spaces, the reader is referred to Lindenstrauss and Tzafriri [8, Volume II, 1.e] or Day [6, Chapter VII, §2]. Convexity in the context of interpolation was studied by Cwikel and Reisner [24] and Vignati [25].

Proposition 5.1. Let $\left\{\mathbf{C}^{n},\|\cdot\|_{z}\right\}_{z \in G}$, where $G \subset \mathbf{C}^{k}$ is bounded (and open) be a (continuous) harmonic interpolation family of normed spaces. If all the spaces $\left\{\mathbf{C}^{n},\|\cdot\|_{\zeta}\right\}_{\zeta \in J G}$ are uniformly convex (cf. [8, Definition 1.e.1(iii)]), then all the intermediate normed spaces $\left\{\mathbf{C}^{n},\|\cdot\|_{z}\right\}_{z \in G}$ are uniformly convex. Quantitatively, if the function $\tilde{\delta}:(0,2] \rightarrow R$ is the largest convex minorant of the moduli of convexity of the boundary spaces $\left(\mathbf{C}^{n},\|\cdot\|_{\zeta}\right) \zeta \in \partial G$, then it is also a minorant of moduli of convexity of the intermediate spaces $\left(\mathbf{C}^{n},\|\cdot\|_{z}\right), z \in G$.

Remark. In the finite-dimensional case, uniform convexity is equivalent to strict convexity. Still, the above formulation has the advantage that it should (presumably) generalize unchanged to the case of infinite-dimensional fibers.

Proof. Denote by $\delta_{z}:(0,2] \rightarrow R$ the modulus of convexity of $\|\cdot\|_{z}$, i.e. $\delta_{z}(\varepsilon)=$ $\inf \left\{1-\frac{1}{2}\|x+y\|_{z}: x, y \in \mathbf{C}^{n},\|x\|_{z}=\|y\|_{z}=1,\|x-y\|_{z}=\varepsilon\right\}, 0<\varepsilon \leq 2$. 
Since $\tilde{\delta}(\varepsilon) \leq \delta_{\zeta}(\varepsilon), \zeta \in \partial G, \varepsilon \in(0,2]$, we get

$$
\frac{1}{2}\|x+y\|_{\zeta}+\tilde{\delta}\left(\|x-y\|_{\zeta}\right) \leq 1, \quad \zeta \in \partial G,\|x\|_{\zeta}=\|y\|_{\zeta}=1 .
$$

By the assumptions, $\tilde{\delta}(\varepsilon)>0$ for $\varepsilon>0$. Fix $\varepsilon_{0}<2$ (rather close to 2). Since the function $\tilde{\delta}$ is convex and positive, one can construct a convex function $\delta^{*}:(0,+\infty) \rightarrow R_{+}$, such that

$$
\begin{aligned}
& \delta^{*}(\varepsilon)=\tilde{\delta}(\varepsilon), \quad \text { for } 0<\varepsilon \leq \varepsilon_{0}, \\
& \delta^{*}(\varepsilon) \leq \tilde{\delta}(\varepsilon), \quad \varepsilon_{0} \leq \varepsilon \leq 2 .
\end{aligned}
$$

Define $u(z, x, y)=\frac{1}{2}\|x+y\|_{z}+\delta^{*}\left(\|x-y\|_{z}\right), z \in \bar{G}, x, y \in \mathbf{C}^{n}$, and $v(z)=$ $\sup \{u(z, x, y): x, y \in B(z)\}$, where $B(z)=\left\{x \in \mathbf{C}^{n}:\|x\|_{z} \leq 1\right\}$. By Remark 2.5(b) and property (1.2), the functions $(z, x, y) \rightarrow\|x+y\|_{z},(z, x, y) \rightarrow$ $\|x-y\|_{z}$ belong to $P_{\text {subh }}\left(G \times \mathbf{C}^{2 n}\right)$; then $\delta^{*}\left(\|x-y\|_{z}\right)$ is of class $P_{\text {subh }}\left(G \times \mathbf{C}^{2 n}\right)$ (note $\delta^{*}$ is convex and increasing; cf. $\left.(1.7)(\mathrm{a})\right)$, and so $u \in P_{\text {subh }}\left(G \times \mathbf{C}^{2 n}\right)$ by (1.1). Since the multifunction $z \rightarrow B(z) \times B(z): G \rightarrow 2^{\mathrm{C}^{n}}$ is harmonic (by Propositions 2.3 and 2.4), $v \in \operatorname{usc}(\bar{G}) \cap \operatorname{subh}(G)$. Hence, $v(z) \leq 1$ for $z \in G$, because $v(\zeta) \leq 1, \zeta \in \partial G$, by $(5.1),(5.2)$ and the definition of $v(\cdot)$. Thus

$$
\frac{1}{2}\|x+y\|_{z}+\tilde{\delta}\left(\|x-y\|_{z}\right) \leq 1, \quad\|x\|_{z},\left\|y_{z}\right\| \leq 1,
$$

provided $\|x-y\|_{z} \leq \varepsilon_{0}$. Since $\varepsilon_{0}$ can be an arbitrary number $<2$, inequality (5.3) holds without further restrictions. Q.E.D.

Corollary 5.2. If $\left\{\mathbf{C}^{n},\|\cdot\|_{z}\right\}_{z \in \bar{G}}$ is a continuous harmonic interpolation family of normed spaces, and all the boundary spaces are uniformly smooth, then so are all the intermediate spaces. Quantitatively, if a convex function $\tilde{\rho}:(0,2) \rightarrow R$, such that $\lim _{\tau \rightarrow 0} \tilde{\rho}(\tau) / \tau=0$, majorizes the moduli of smoothness of all the boundary spaces, it also majorizes the moduli of smoothness of all the intermediate spaces.

This follows directly from the previous proposition applied to the family of dual normed spaces (cf. Theorem 2.9) and from the fact that the lower convex envelope of the modulus of convexity of a given normed space is the convexconjugate function of the module of smoothness of its dual normed spaces, (cf. [8, Propositions 1.e.2 and 1.e.6]).

Unlike the modulus of convexity, the modulus of smoothness makes sense also for quasinormed spaces. If $p: X \rightarrow R$ is a quasinorm, the modulus of smoothness of the space $(X, p)$ is

$$
\rho_{X}(t)=\sup \{(p(x+h)+p(x-h)) / 2-1: x, h \in X, p(x)=1, p(h)=t\} .
$$

The next lemma will yield new applications also in the case of normed spaces.

Lemma 5.3. Let $\left\{\mathbf{C}^{n}, p_{z}(\cdot)\right\}_{z \in G}$, where $G \subset \mathbf{C}^{k}$ is open, be a harmonic interpolation family of quasinormed spaces. Denote

$$
\rho(z, t)=\sup \left\{\frac{1}{2}\left(p_{z}(x+h)+p_{z}(x-h)\right)-1: x, h \in \mathbf{C}^{n}, p_{z}(x)=1, p_{z}(h)=t\right\}
$$


for $t \geq 0$. Then the function $z \rightarrow \rho\left(z, e^{h(z)}\right): G_{0} \rightarrow R$ is a subharmonic function for every harmonic function $h: G_{0} \rightarrow R$, where $G_{0} \subset G$.

Proof. Clearly, $\rho: G \times \mathbf{C}^{n} \rightarrow R$ is a usc function. (If all the quasinorms $p_{z}(\cdot)$ are actually norms, then $\rho(z, \tau)$ is continuous.)

Assertion. Let

$$
\chi(z, w)=\max \left\{\frac{1}{2} p_{z}\left(x+e^{i \theta} w\right)+\frac{1}{2} p_{z}\left(x-e^{i \theta} w\right)-1: p_{z}(x)=1, \theta \in R\right\}
$$

for $(z, w) \in G \times \mathbf{C}^{n}$. Then $\chi \in P_{\text {subh }}\left(G \times \mathbf{C}^{n}\right)$.

To prove the assertion, let, for $(z, x, w) \in G \times \mathbf{C}^{n} \times \mathbf{C}^{n}$,

$$
u(z, x, w)=\max \left\{\frac{1}{2} p_{z}\left(x+e^{i \theta} w\right)+\frac{1}{2} p_{z}\left(x-e^{i \theta} w\right)-1: \theta \in R\right\} .
$$

Then $u \in P_{\text {subh }}\left(G \times \mathbf{C}^{2 n}\right)$, by (1.2), (1.1), and the fact that the supremum of an upper-bounded family of $P_{\text {subh }}$ functions is $P_{\text {subh }}$, provided it is usc. Since the quasinorms $p_{z}(\cdot)$ are $\mathbf{C}$-homogeneous,

$$
\begin{aligned}
u(z, r x, y) & =\max _{\theta \in R}\left(\frac{1}{2} p_{z}\left(r e^{-i \theta} x+w\right)+\frac{1}{2} p_{z}\left(r e^{-i \theta} x-w\right)-1\right) \\
& =\max \left\{\frac{1}{2} p_{z}(\alpha x+w)+\frac{1}{2} p_{z}(\alpha x-w)-1:|\alpha|=r, \alpha \in \mathbf{C}\right\} .
\end{aligned}
$$

Since $\alpha \rightarrow P_{z}(\alpha x \pm w)$ is a subharmonic function, we conclude that $u(z, r x, w)$ $\leq u(z, x, w)$ if $0 \leq r \leq 1$. Thus $\chi(z, w)=\max \{u(z, x, w): x \in B(z)\}$, where $B(z)=\left\{x \in \mathbf{C}^{n}: p_{z}(x) \leq 1\right\}$, and so $\chi \in P_{\text {subh }}\left(G \times \mathbf{C}^{n}\right)$, by Proposition 2.4 and Definition 2.1. This proves the assertion.

Let $W(z)=e^{h(z)} B(z)=\left\{e^{h(z)} w: w \in B(z)\right\}$. Since $B(\cdot)$ is a harmonic multifunction, and $B(z), z \in G$, are unit balls of $\mathbf{C}$-homogeneous quasinorms, Propositions 2.4 and 3.4 imply that $z \rightarrow W(z): G_{0} \rightarrow 2^{\mathrm{C}^{n}}$ is a harmonic multifunction, provided $h: G_{0} \rightarrow R$ is a harmonic function. Finally, $\rho\left(z, e^{h(z)}\right)=\max \{\chi(z, w): w \in W(z)\}, z \in G_{0}$. The last observations and the assertion imply that $z \rightarrow \rho\left(z, e^{h(z)}\right)$ is a subharmonic function in $G_{0}$. Q.E.D.

The most important class of uniformly smooth spaces form those whose modulus of smoothness is of power type $q$, with $q \in(1,2]$, i.e. such that for some $K<+\infty, \rho_{X}(t) \leq K t^{q}, t>0$.

Theorem 5.4. Let $\left\{\mathbf{C}^{n}, p_{z}(\cdot)\right\}_{z \in \bar{G}}$, where $G \subset \mathbf{C}^{k}$ is bounded, be a continuous harmonic interpolation family of quasinormed spaces. Assume that all the boundary spaces have moduli of smoothness of power-type $q_{0} \in(1,2]$ with a uniform constant $K_{0}$, i.e. $\rho(\zeta, t) \leq K_{0} t^{q}, \zeta \in \partial G, t \geq 0$. Then every intermediate space $\left(\mathbf{C}^{n}, p_{z}(\cdot)\right), \quad z \in G$, has modulus of smoothness of power type $q_{0}$. Moreover, if $\gamma(z)=$ the supremum of $q$ 's, such that $p_{z}(\cdot)$ has modulus of smoothness of power type $q$, then the function $z \rightarrow 1 / \gamma(z): G \rightarrow R$ is a bounded, Borel-measurable, weak subharmonic function.

Proof. By Lemma 5.3, the function $z \rightarrow \rho(z, t)$ is subharmonic for every $t \geq 0$; hence $\rho(z, t) \leq K_{0} t^{q_{0}}, z \in G$. For $K \geq K_{0}$, let

$$
\gamma(K, z)=\max \left\{q \in[1,2]: \rho(z, t) \leq K t^{q}, 0 \leq t<+\infty\right\} .
$$


Assertion 1. If $K \geq K_{0}$, then the function $z \rightarrow 1 / \gamma(K, z)$ is 1sc in $G$ and satisfies the mean-value inequality.

This assertion implies the theorem. Indeed, if $K_{0} \leq K_{1} \leq K_{2}$, then the set $\left\{q \in[1,2]: \rho(z, t) \leq K_{1} t^{q}, t \in(0,+\infty)\right\}$ is contained in $\{q: q \in[1,2]: \rho(z, t)$ $\left.\leq K_{2} t^{q}, t \in(0,+\infty)\right\}$, and so $\gamma\left(K_{1}, t\right) \leq \gamma\left(K_{2}, t\right)$. Hence, $1 / \gamma(n, z) \searrow 1 / \gamma(z)$, $z \in G$. Since the function $1 / \gamma(n, z), n=1,2, \ldots$, form a uniformly bounded family of lsc functions, and since they satisfy the mean-value inequality (by Assertion 1), $1 / \gamma(z)$ is weak subharmonic.

As for Assertion 1, observe that $\gamma(K, z)=\max \{q: q \in A(z)\}$, where $q \in$ $A(z)$, if and only if $1 \leq q \leq 2$ and $\frac{1}{2} p_{z}(x+h)+\frac{1}{2} p_{z}(x-h)-1 \leq K p_{z}(h)$, whenever $p_{z}(x) \leq 1, x, h \in \mathbf{C}^{n}$. Since $(z, x) \rightarrow p_{z}(x): \bar{G} \times \mathbf{C}^{n} \rightarrow R$ is a continuous function, the multifunction $z \rightarrow A(z): \bar{G} \rightarrow 2^{[1,2]}$ is usc and compact-valued, and so $z \rightarrow \gamma(K, z)$ is a usc function for every $K \geq K_{0}$.

To prove the mean-value inequality, it suffices to check that whenever $B_{0}$ is a Euclidean ball with $\bar{B}_{0} \subset G$ and $h: B_{0} \rightarrow R$ is a bounded harmonic function (note that $1 / \gamma(K, z)$ is bounded), such that $\tilde{h}(\zeta)>1 / \gamma(K, \zeta)$ for a.a. $\zeta \in \partial B_{0}$, where $\tilde{h}(\zeta)$ denote the nontangential boundary values, then $h(z) \geq 1 / \gamma(K, z)$, $z \in B_{0}$.

Let $\zeta$ be any point of $\partial B_{0}$ at which the nontangential boundary limit exists. Since $1 / \tilde{h}(\zeta)<\gamma(K, \zeta)$, we get $\rho(\zeta, \tau) \leq K \tau^{1 / \hat{h}(\zeta)}$, or equivalently $\rho\left(\zeta, t^{\hat{h}(\zeta)}\right) \leq$ $K t$ for $t>0$. Fix now $t>0$ and let $\varphi(z)=\rho\left(z, t^{h(z)}\right), z \in B_{0}$. By Lemma 5.3 , function $\varphi(\cdot)$ is subharmonic in $B_{0}$. Since $\rho(z, \tau)$ is usc, one can see that, if the nontangential boundary value $\tilde{h}(\zeta)$ exists at the point $\zeta \in \partial B_{0}$, then

$$
(\text { nontan }) \limsup _{z \rightarrow \zeta} \varphi(z) \leq \rho\left(\zeta, t^{\grave{h}(\zeta)}\right) \leq K t,
$$

where $z$ approaches $\zeta$ nontangentially in lim sup.

The following fact is well known.

Assertion 2. Let $\varphi: B_{0} \rightarrow R$ be a bounded subharmonic function and $C$ a constant. Assume that $\limsup _{r \rightarrow 1-0} \varphi(r \zeta) \leq C$ for a.a. $\zeta \in \partial B_{0}$. Then $\varphi(z) \leq$ $C$ for all $z \in B_{0}$.

By (5.4) and Assertion 2, $\varphi(z)=\rho\left(z, t^{h(z)}\right) \leq K t$ for $z \in B_{0}$ and $t>0$. Hence, $\rho(z, \tau) \leq K \tau^{1 / h(z)}, \tau>0$, which means that $1 / h(z) \leq \gamma(K, z)$, i.e. $1 / \gamma(K, z) \leq h(z), z \in B_{0}$. Q.E.D.

Recall, after Lindenstrauss and Tzafriri $[8, \S 1 . e]$, that a normed space $\left(\mathbf{C}^{n},\|\cdot\|_{z}\right)$ has modulus of convexity $\delta_{z}(\varepsilon)$ of power type $p \in(1,2]$, if $\delta_{z}(\varepsilon) \geq c \varepsilon^{p}, \varepsilon \in(0,2]$, with some positive constant $c$. By [8, Proposition 1.e.2], the normed space $\left(\mathbf{C}^{n},\|\cdot\|_{z}\right)$ has modulus of convexity of power type $p$ if and only if the dual normed space has modulus of smoothness of power type $q$, where $1 / p+1 / q=1,2 \geq p, q \geq 1$.

Remark 5.5. By the above observations and the duality for harmonic interpolation (Theorem 2.9), Theorem 5.4 has an obvious analog for uniform convexity. 
In particular, if the normed spaces $\left(\mathbf{C}^{n},\|\cdot\|_{z}\right), z \in G$, form a harmonic interpolation family and have modulus of convexity of power type $p_{0}>1$ (with uniform constant $c>0)$, then $z \rightarrow 1 / \pi(z): G \rightarrow R$ is a superharmonic function, where $\pi(z)=$ the infimum of admissible exponents $p$ of power type for the space $\left(\mathbf{C}^{n},\|\cdot\|_{z}\right)$.

Proof of Theorem 2.10. Let

$$
R(z)=\left\{r \in[0,1]: p_{z}(x+y)^{r} \leq p_{z}(x)^{r}+p_{z}(y)^{r}, x, y \in \mathbf{C}^{n}\right\} .
$$

Clearly, the multifunction $z \rightarrow R(z): G \rightarrow 2^{[0,1]}$ is usc and compact-valued and $r(z)=\max \{r: r \in R(z)\}$. Thus $r(\cdot)$ is a usc function and $z \rightarrow 1 / r(z)$ is lsc, as required.

The general idea of the proof of the mean-value inequality is similar to the proof of Theorem 5.4. Since $1 / r(z)$ is a bounded function, it suffices to show that for every bounded harmonic function $h: B_{0} \rightarrow R$, with $B_{0}$ a Euclidean ball and $\bar{B}_{0} \subset G$, such that $\tilde{h}(\zeta) \geq 1 / r(\zeta)$ for a.a. $\zeta \in \partial B_{0}(\tilde{h}(\zeta)$ stands for the nontangential boundary value), the inequality $h(z) \geq 1 / r(z), z \in B_{0}$, must hold.

Fix such a $B_{0}$ and $h$ and denote by $A$ the set of all those $\zeta \in \partial B_{0}$ at which the nontangential boundary limit $\tilde{h}(\zeta)$ exists and $\tilde{h}(\zeta) \geq 1 / r(\zeta)$. Define the multifunction $K: B_{0} \cup A \rightarrow 2^{\mathrm{C}^{m}}$ ( $m$ is a fixed integer) by formula (3.1) if $z \in B_{0}$, and let

$$
K(\zeta)=\left\{w \in \mathbf{C}^{m}: \sum_{i=1}^{m}\left|w_{i}\right|^{1 / \hat{h}(\zeta)} \leq 1\right\} \quad \text { if } \zeta \in A .
$$

Let $B(z)=\left\{x \in \mathbf{C}^{n}: p_{z}(x) \leq 1\right\}$. For $\zeta \in A$, we have $1 / \tilde{h}(\zeta) \leq r(\zeta)$, and so the function $x \rightarrow\left(p_{\zeta}(x)\right)^{1 / \hat{h}(\zeta)}$ is subadditive and $1 / \tilde{h}(\zeta)$-homogeneous on $\mathbf{C}^{n}$. Thus

$$
w_{1} x_{1}+\cdots+w_{m} x_{m} \in B(\zeta) \text { if } x_{1}, \ldots, x_{m} \in B(\zeta), w \in K(\zeta), \zeta \in A .
$$

Denote $F\left(w, x_{1}, \ldots, x_{m}\right)=w_{1} x_{1}+\cdots+w_{m} x_{m}$ for $w \in \mathbf{C}^{m}, x_{i} \in \mathbf{C}^{n}, i=$ $1, \ldots, m$, and let $R(z)=F\left(K(z) \times B(z)^{m}\right)$ for $z \in B_{0} \cup A$, where $B(z)^{m}$ stands for the $m$-fold Cartesian product $B(z) \times \cdots \times B(z)$. Since $F$ is an analytic mapping, the multifunction $z \rightarrow R(z): B_{0} \rightarrow 2^{\mathrm{C}^{n}}$ is harmonic by Propositions 2.3 and 3.2 .

Now let $\varphi(z)=\max \left\{p_{z}(x): x \in R(z)\right\}, z \in B_{0} \cup A$. Since $R \mid B_{0}$ is a harmonic multifunction, $\varphi \mid B_{0}$ is a subharmonic function. By $(5.5), R(\zeta) \subset B(\zeta)$ for $\zeta \in A$, and so $\varphi(\zeta) \leq 1$ for $\zeta \in A$. Since $h(z) \rightarrow \tilde{h}(\zeta)$, as $z \rightarrow \zeta$ nontangentially (for a fixed $\zeta \in A$ ), $\operatorname{Lim} \operatorname{Sup} R(z) \subset R(\zeta)$ if $z \rightarrow \zeta$ nontangentially (Lim Sup means in the topological, not set-theoretical, sense), and the function $(z, x) \rightarrow p_{z}(x)$ is continuous, then $\lim \sup \varphi(z) \leq \varphi(\zeta) \leq 1$, provided $z \rightarrow \zeta$ nontangentially (for $\zeta \in A$ ). Since $A$ has full measure, Assertion 2 in the Proof of Theorem 5.4 implies that $\varphi(z) \leq 1$ for $z \in B_{0}$, and so $R(z) \subset B(z)$, 
$z \in B_{0}$. This inclusion means that $w_{1} x_{1}+\cdots+w_{m} x_{m} \in B(z)$, whenever $\sum_{i=1}^{m}\left|w_{i}\right|^{1 / h(z)} \leq 1$, and $x_{1}, \ldots, x_{m} \in B(z)$. Since $m$ is arbitrary, we conclude that the function $x \rightarrow p_{z}(x)^{1 / h(z)}$ satisfies the triangle inequality for $z \in B_{0}$. Thus $1 / h(z) \leq r(z)$. Q.E.D.

\section{COMPARISON OF HARMONIC INTERPOLATION WITH OTHER COMPLEX INTERPOLATION METHODS}

As mentioned in the introduction, the harmonic interpolation method is just a special case of the $P_{Q}^{\text {psh }}$-interpolation method (or $P_{Q}^{\text {co }}$ if we want to obtain normed spaces), where $Q$ is an arbitrary translation invariant complex pseudoconvex class of usc functions, with local maximum properties on $\mathbf{C}^{k}$ (or, more generally, on a homogeneous complex manifold). For the list of properties of $Q$ and the construction of interpolating quasinorms, the reader is referred to [18, $\S \S 1,2]$. We recall, however, that $P_{Q}^{\text {psh }}$ is the class of all usc functions $u(z, w)$ defined (on open subsets) of $\mathbf{C}^{k} \times \mathbf{C}^{n}$, such that the function $z \rightarrow u(z, f(z))$ is of class $Q$ if $z \rightarrow f(z): G_{0} \subset \mathbf{C}^{n}, G_{0} \subset \mathbf{C}^{k}$, is analytic and the slice functions $w \rightarrow u(z, w)$ are plurisubharmonic.

As indicated in the introduction, in order to understand better the remarkable regularity of the harmonic interpolation method, we compare it in this section with other $P_{Q}^{\mathrm{psh}}$-methods (or $P_{Q}^{\mathrm{co}}$-methods), in case $Q+Q \subset Q$, or $Q^{d}+Q^{d} \subset$ $Q^{d}$ (cf. Terminology 1.1 for the definition of $Q^{d}$ ).

The additivity of $Q$ is applied through the following property:

$$
\text { if } Q+Q \subset Q \text {, then } P_{Q}^{\mathrm{psh}}+P_{Q}^{\mathrm{psh}} \subset P_{Q}^{\mathrm{psh}} \text {. }
$$

In this respect recall that (cf. [18, Theorem 4.1])

a family $\left\{\mathbf{C}^{n}, p_{z}(\cdot)\right\}_{z \in G}$ of quasinormed spaces is a $P_{Q}^{\text {psh }}$-subinterpolation family if and only if $(z, w) \rightarrow \log p_{z}(w)$ is a function of class $P_{Q}^{\mathrm{psh}}\left(G \times \mathbf{C}^{n}\right)$.

Property (6.1) is used in those proofs in which the maximum property for sums of functions built from the norm function is required.

As for the additivity of $Q^{d}$, the main reason of its usefulness for our applications is comprised by the next theorem.

Theorem 6.1. Let $\left\{\mathbf{C}^{n}, p_{z}(\cdot)\right\}_{z \in \bar{G}}$, with $G \subset \mathbf{C}^{k}$ bounded, be a $P_{Q}^{\mathrm{psh}}$-interpolation family of quasinormed spaces. Let $B(z)=\left\{w \in \mathbf{C}^{n}: p_{z}(w) \leq 1\right\}$. Then the set $\left\{\left(z, w_{1}, w_{2}\right) \in G \times \mathbf{C}^{n} \times \mathbf{C}^{n}: w_{1}, w_{2} \in B(z)\right\}$ has local maximum property with respect to functions $u\left(z, w_{1}, w_{2}\right)$ of class $P_{Q}^{\mathrm{psh}}$ on $G \times \mathbf{C}^{2 n}$.

In fact, spaces $\mathbf{C}^{2 n}$ with quasinorms determined by $W(z) \times W(z), z \in G$, form a $P_{Q}^{\text {psh }}$-interpolation family (provided $Q^{d}+Q^{d} \subset Q^{d}$ ), which generalizes partially Proposition 4.6 above. 
Instead of using Theorem 6.1 directly, it is actually more convenient to use, like in $\S \S 2-5$, some auxiliary classes of set-valued functions.

Definition 6.2. Let $R$ be a pseudoconvex class of functions on $\mathbf{C}^{k}$ such that $R+R \subset R$ and $R$ contains the class of plurisubharmonic functions (cf. [18, (1.1)-(1.2)]). Then a multifunction $z \rightarrow K(z): G \rightarrow 2^{\mathbf{C}^{n}}, G \subset \mathbf{C}^{k}$, is called weak $R$-analytic if it is compact valued, usc, and satisfies the condition:

$$
\begin{aligned}
& \text { for every integer } s=0,1,2, \ldots \text { and for every function } \\
& u(z, w, \xi) \text { of class } P_{R}^{\text {psh }} \text { on } H \subset G \times \mathbf{C}^{n+s} \text {, the function } \\
& u(z, \xi)=\max \{u(z, w, \xi): w \in K(z)\} \text { is of class } P_{R}^{\text {psh on }} \\
& \{(z, \xi): z \in G,\{z\} \times K(z) \times\{\xi\} \subset H\} .
\end{aligned}
$$

Examples. If $R=$ subh, weak $R$-analytic multifunctions $=$ harmonic multifunctions. If $R=\mathrm{psh}$, weak $R$-analaytic multifunctions $=$ weak analytic multifunctions (cf. [15, Definition 3.2; 11, §4]).

Proposition 6.3. If $z \rightarrow K(z): G \rightarrow 2^{\mathrm{C}^{n}}$ is a usc multifunction with convex sections whose graph has local maximum property with respect to the class $P_{Q}^{\mathrm{psh}}$ on $G \times \mathbf{C}^{n}$, and $Q^{d}+Q^{d} \subset Q^{d}$, then $z \rightarrow K(z)$ is a weak $Q^{d}$-analytic multifunction. In particular, the multifunction $z \rightarrow B(z): G \rightarrow 2^{\mathrm{C}^{n}}$ from Theorem 6.1 is weak $Q^{d}$-analytic.

Proposition 6.4. Let $z \rightarrow K(z): G \rightarrow 2^{\mathrm{C}^{n}}$ and $z \rightarrow L(z): G \rightarrow 2^{\mathrm{C}^{m}}$ be weak $R$-analytic multifunctions (as in Definition 6.2). Then the multifunction $z \rightarrow$ $K(z) \times L(z): G \rightarrow 2^{\mathrm{C}^{n+m}}$ is weak $R$-analytic.

The first proposition summarizes [18, Proposition 1.3, Theorem 3.5]. The proof of Proposition 6.4 is completely analogous to that of Proposition 2.3. (In fact the proof in $\S 2$ was axiomatic: the specific way in which classes of the function on $G \times \mathbf{C}^{n} \times \mathbf{C}^{s}, s=0,1,2, \ldots$, were defined, was never used.)

We will sketch now generalizations of some of the results of $\S \S 2$ and 5 , in which the additivity of $Q^{d}$ is used.

Proposition 6.5. Let $Q$ be a translation invariant pseudoconvex class of functions on $\mathbf{C}^{k}$, containing the class of plurisubharmonic functions and having local maximum property (cf. [18, Axioms (1.1)-(1.12)]). Assume that $Q^{d}+Q^{d} \subset Q^{d}$ and let $\left\{\mathbf{C}^{n}, p_{z}(\cdot)\right\}_{z \in G}$ be a continuous $P_{Q}^{\mathrm{psh}}$-interpolation family of quasinormed spaces. Then:

(a) if all the boundary quasinorms $p_{\zeta}, \zeta \in \partial G$, are norms, then all $p_{z}$, $z \in G$, are norms as well;

(b) in the situation of $(\mathbf{a}),\left\{\mathbf{C}^{n}, p_{z}(\cdot)\right\}_{z \in \bar{G}}$ is also a continuous $P_{Q}^{\mathrm{co}}$-interpolation family (of normed spaces), i.e. $P_{Q}^{\mathrm{co}}$ - and $P_{Q}^{\mathrm{psh}}$-interpolation methods yield identical results when the boundary data are normed spaces; 
(c) if $r \in(0,1]$ and all the $p$ 's, $\zeta \in \partial G$, are $r$-norms, i.e. $p_{\zeta}(x)^{r}$ satisfies the triangle inequality, then so are all the intermediate quasinorms $p_{z}, z \in G$. Proof (sketch). (a) is a special case of (c) with $r=1$.

(c) We proceed as in Lemma 2.6. To obtain that $p_{z}(x+y)^{r} \leq p_{z}(x)^{r}+p_{z}(y)^{r}$, $x, y \in \mathbf{C}^{n}$, it suffices to show, for every $\alpha, \beta>0$, that $p_{z}(\alpha x+\beta y)^{r} \leq \alpha^{r}+\beta^{r}$ if $p_{z}(x) \leq 1, p_{z}(y) \leq 1$. This is equivalent to proving that $v(z) \leq 1$ for $z \in G$, where

$$
v(z)=\max \{u(z, x, y):(x, y) \in W(z) \times W(z)\}
$$

and $u(z, x, y)=p_{z}(\alpha x+\beta y)^{r}, W(z)=\left\{w \in \mathbf{C}^{n}: p_{z}(w) \leq 1\right\}$. Using [18, Theorem 4.1], the definition of the class $P_{Q^{d}}^{\mathrm{psh}}$ (cf. [19, §4] and a property analogous to $(1.7))$, one checks easily that $u \in P_{Q^{d}}^{\text {psh }}\left(G \times \mathbf{C}^{2 n}\right)$. Now, by Propositions 6.3 and 6.4, $v \in Q^{d}(G) \cap$ usc $(\bar{G})$. Since the class $Q^{d}$ has the local maximum property (cf. [17, Proposition 1.12(i)]), $v(z) \leq \max v \mid \partial G$, and so $v(z) \leq \alpha^{r}+\beta^{r}$, because $v(\zeta) \leq \alpha^{r}+\beta^{r}, \zeta \in \partial G$, by the subadditivity property of $p^{r}(\zeta)$.

(b) follows from (a) and the next Assertion.

Assertion. Let $Q$ satisfy conditions (1.0)-(1.12) in [18] and let $\left\{\mathbf{C}^{n}, p_{z}\right\}_{z \in \bar{G}}$ be a $P_{Q}^{\text {psh }}$-interpolation family. Assume that all the $p_{z}(\cdot), z \in G$, are norms. Then $\left\{\mathbf{C}^{n}, p_{z}(\cdot)\right\}_{z \in \bar{G}}$ is also a $P_{Q}^{\text {co }}$-interpolation family.

Although no additivity assumption is made about $Q$ or $Q^{d}$, the proof of this assertion is practically identical with the Proposition 2.8 above, and is omitted. Q.E.D.

Theorem 6.6. In the situation of Proposition 6.5(c), let $r(z)=$ the largest $r>0$, such that the function $w \rightarrow p_{z}(w)^{r}$ satisfies the triangle inequality. Then the usc regularization of the function $z \rightarrow 1 / r(z)$ is $(k-1)$-plurisubharmonic. (More precisely, it is of class $Q$.)

Proof (sketch). The proof is very similar to that of Theorem 2.10 (and slightly simpler, because the nontangential limits do not have to be considered). Keeping the notation of the latter proof, we have to introduce the following changes.

Function $h: \bar{B}_{0} \rightarrow R$ is now assumed continuous and $-h \in Q^{d}$. Then the definition of $K(z)$ is literally the same, but the conclusion is now that $K: B_{0} \rightarrow 2^{\mathrm{C}^{m}}$ is a $Q^{d}$-analytic multifunction (which one obtains by [18, Proof of Lemma 2.8, Assertion 1], and so is the multifunction $R(\cdot)$, by Proposition 6.4 and an analog of Proposition 3.2 for weak $Q^{d}$-analytic multifunctions. We omit further details. Q.E.D.

We will consider now some properties of $P_{Q}^{\text {psh }}$-interpolation method in the situation when $Q+Q \subset Q$. We start from a counterexample.

Proposition-Example 6.7. For $K>0$, let $X=\left\{(\zeta, w) \in \mathbf{C}^{2} \times \mathbf{C}^{2}:|\zeta|=1,\left|w_{i}\right| \leq\right.$ $\left.e^{-K\left|\zeta_{i}\right|^{2}}, i=1,2\right\}$. Then the polynomial hull of $X$ is equal to the set

$$
Y=\left\{(z, w) \in \mathbf{C}^{2} \times \mathbf{C}^{2}:|z| \leq 1,\left|w_{1} w_{2}\right| \leq e^{-K},\left|w_{i}\right| \leq e^{-K\left|z_{i}\right|^{2}}, i=1,2\right\} .
$$


Proof. Observe that $Y$ contains $X$ and is equal to the intersection of the following three sets:

$$
\begin{aligned}
& \left\{(z, w) \in \mathbf{C}^{4}:|z| \leq 1,\left|w_{1} w_{2}\right| \leq e^{-K}\right\} ; \\
& \left\{\left(z, w_{1}\right) \in \mathbf{C}^{3}:|z| \leq 1,\left|w_{1}\right| \leq e^{-K\left|z_{1}\right|^{2}}\right\} \times \bar{D} ; \\
& \left\{\left(z, w_{2}\right) \in \mathbf{C}^{3}:|z| \leq 1,\left|w_{2}\right| \leq e^{-K\left|z_{2}\right|^{2}}\right\} \times \bar{D},
\end{aligned}
$$

where $D$ denotes the closed unit disc. Clearly, the first set is polynomially convex; the other two sets are polynomially convex because, in general, the sets of the form $\left\{(z, w) \in \mathbf{C}^{3}:|z| \leq 1,|w| \leq e^{-\varphi(z)}\right\}$, where $\varphi(z)$ is continuous and plurisubharmonic, are known to be polynomially convex (Hartogs sets). Hence, $Y \supset \operatorname{hull}(X)$.

Denote $\partial B=\left\{z \in \mathbf{C}^{2}:|z|=1\right\}$. Since $Y \cap\left(\partial B \times \mathbf{C}^{2}\right)=X \cap\left(\partial B \times \mathbf{C}^{2}\right)$, it remains to show (by Rossi's local maximum modulus principle) that the set $Y_{1}=Y \backslash\left(\partial B \times \mathbf{C}^{2}\right)$ has local maximum property (relative to polynomials). For this, it suffices to check that through each point $(a, b)$ of $\partial Y \cap\left(B \times \mathbf{C}^{2}\right)$ (where $\left.B=\left\{z \in \mathbf{C}^{2}:|z|<1\right\}\right)$, there passes an open piece of one-dimensional complex variety, entirely contained in $Y$.

If $(a, b) \in \partial Y$ and $|a|<1$, then one of the following three cases holds:

$$
\begin{aligned}
& \left|b_{1}\right|=e^{-K\left|a_{1}\right|^{2}}, \quad\left|b_{1} b_{2}\right| \leq e^{-K} ; \\
& \left|b_{2}\right|=e^{-K\left|a_{2}\right|^{2}}, \quad\left|b_{1} b_{2}\right| \leq e^{-K} ; \\
& \left|b_{i}\right|<e^{-K\left|a_{i}\right|^{2}}, \quad i=1,2, \quad\left|b_{1} b_{2}\right|=e^{-K} .
\end{aligned}
$$

In the third case, we just take an open piece of the 2-plane $\{(z, w): w=b\}$. The first two cases are symmetric and we consider only the first of them. There is an open neighborhood of $(a, b)$ in the complex lines $\left\{\left(a, z_{2}, b_{1}, b_{2}\right): z_{2} \in \mathbf{C}\right\}$ which is contained in $Y$, because the conditions $|a|<1,\left|b_{1} b_{2}\right| \leq e^{-K}$ and $\left|b_{1}\right|=e^{-K\left|a_{1}\right|^{2}}$ imply necessarily that $\left|b_{2}\right|<e^{-K\left|a_{2}\right|^{2}}$, and so $\left|b_{2}\right|<e^{-K\left|z_{2}\right|^{2}}$ for $z_{2}$ near $a_{2}$. Q.E.D.

We can show now that the assumption $Q^{d}+Q^{d} \subset Q^{d}$ cannot be omitted from Proposition 6.5(a).

Corollary 6.8. If $Q$ is the class of all plurisubharmonic functions on $\mathbf{C}^{2}$, then $P_{Q}^{\mathrm{co}}$ - and $P_{Q}^{\mathrm{psh}}$-interpolation methods yield different results for some continuous family of normed spaces on $\partial B=\left\{|\zeta|=1, \zeta \in \mathbf{C}^{2}\right\}$.

Proof. Let $p_{\zeta}(w)=\max \left(e^{K\left|\zeta_{1}\right|^{2}}\left|w_{1}\right|, e^{K\left|\zeta_{2}\right|^{2}}\left|w_{2}\right|\right), w \in \mathbf{C}^{2}, \zeta \in \partial B$. Then $\left\{(\zeta, w) \in \mathbf{C}^{4}:|\zeta|=1, p_{\zeta}(w) \leq 1\right\}$ is equal to the set $X$ from the last example. It is easy to observe (cf. [19, Proposition 4.7]) that if $Q=$ psh (on $\mathbf{C}^{2}$ ), then $P_{Q}^{\mathrm{psh}}\left(\right.$ on $\left.\mathbf{C}^{2} \times \mathbf{C}^{2}\right)=$ psh $\left(\right.$ on $\left.\mathbf{C}^{4}\right)$.

By [18, Definition 2.1], if $\tilde{p}_{z}(\cdot), z \in G$, is the family of interpolating quasinorms, relative to $P_{Q}^{\mathrm{psh}}=\mathrm{psh}$, and $W(z)=\left\{w: p_{z}(w) \leq 1\right\}$, then $\operatorname{gr}(W) \backslash X=$ 
the largest set with local maximum property with respect to psh (and compact closure), and so is equal to $Y \backslash X$ (in the notation of Proposition-Example 6.7). By the properties of $Y, W(z)$ is not convex for $|z|<1$, i.e. $p_{z}(\cdot)$ are not norms. Q.E.D.

Remark. One may ask whether an example of a polynomial hull with the properties of $Y$ from Proposition-Example 6.7 can be found in a space of lower dimension than four. If one drops the requirement that the sections of $Y$ be circled, then the three-dimensional example due to John Wermer [22] (which we sketch below with its author permission) provides a positive answer. On the other hand, no such example is possible if $B$ is replaced by a planar compact (cf. Alexander and Werner [1] for the case of the unit disc, and [16]).

Example 6.9 [22]. The sets $R_{i}=\left\{\zeta \in \partial B:\left|\zeta_{i}\right|<\frac{1}{2}\right\}, i=1,2$, are open subsets of the sphere $\partial B$ and $\bar{R}_{1} \cap \bar{R}_{2}=\varnothing$. Using Tietze-Urysohn's theorem, one can construct easily a continuous family of closed circles $\zeta \rightarrow X_{\zeta}: \partial B \rightarrow 2^{\mathrm{C}}$, such that:

(a) $X_{\zeta}=\{|w+2| \leq 1\}$ if $\zeta_{1}=0, \zeta \in \partial B$, and $X_{\zeta}=\{|w-2| \leq 1\}$ if $\zeta_{2}=0, \zeta \in \partial B$

(b) $X_{\zeta} \subset\{|w+2| \leq 1\}$ if $\zeta \in R_{1}$ and $X_{\zeta} \in\{|w-2| \leq 1\}$ if $\zeta \in R_{2}$;

(c) if $\zeta \in \partial B \backslash\left(R_{1} \cup R_{2}\right)$, then $X_{\zeta}$ is a singleton $\{t\}$, with $t \in[-2,2]$.

We now let $X=\left\{(\zeta, w) \in \partial B \times \mathbf{C}: w \in X_{\zeta}\right\}$, and denote the polynomial hull of $X$ by $Y$. By the well-known facts, $Y \cap(\partial B \times \mathbf{C})=X$. Denote $Y_{0}=\{w \in \mathbf{C}:(0, w) \in Y\}$. Since the set

$$
Z=\bigcup_{\zeta \in \partial B} X_{\zeta}=\{|w+2| \leq 1\} \cup[-1,1] \cup\{|w-2| \leq 1\}
$$

is a polynomially convex planar set, $Y_{0} \subset Z$. Since $X_{\zeta}$ is constant on each of the circles $\left\{\zeta \in \partial B: \zeta_{i}=0\right\}, i=1,2, Y_{0}$ must contain both discs $\{|w+2| \leq$ $1\}$ and $\{|w-2| \leq 1\}$. Since $Z$ is not (geometrically) convex, we conclude that $Y_{0}$ cannot be convex either.

Theorem 6.10. Let $Q$ be a translation-invariant pseudoconvex class on $\mathbf{C}^{k}$ with local maximum property (cf. [18, (1.1)-(1.12)]). Let $\left\{\mathbf{C}^{n}, p_{z}(\cdot)\right\}_{z \in \bar{G}}$ be a $P_{Q}^{\mathrm{co}}$ (or $P_{Q}^{\mathrm{psh}}{ }^{-}$) interpolation family of normed (resp. quasinormed) spaces (on a bounded set $\bar{G})$. Assume that $Q$ is closed with respect to addition. Then

(a) if there is $K>0$, such that

$$
p_{\zeta}(x+h)^{2}+p_{\zeta}(x-h)^{2} \leq 2 p(x)^{2}+K|h|^{2}, \quad x, h \in \mathbf{C}^{n}, \zeta \in \partial G,
$$

then for every $z \in \bar{G}, x, h \in \mathbf{C}^{n}$

$$
p_{z}(x+h)^{2}+p_{z}(x-h)^{2} \leq 2 p_{z}(x)^{2}+K|h|^{2},
$$

where $|\cdot|$ denotes the Euclidean norm; 
(b) if the functions $w \rightarrow p_{\zeta}(w)^{2}$ have $L^{\infty}$ second order partial derivatives for every $\zeta \in \partial G$, and if they are uniformly bounded in $\zeta \in \partial G$, then the function $(z, w) \rightarrow p_{z}(w)^{2}$ has $L^{\infty}$ second order partial derivatives with respect to $w$ in $G \times \mathbf{C}^{n}$.

Remark. In case $Q=$ psh, the theorem has an interpretation in terms of polynomial hulls. If we let $X=\left\{(\zeta, w) \in \mathbf{C}^{k} \times \mathbf{C}^{n}: \zeta \in \partial G, p_{\zeta}(w) \leq 1\right\}$ and $Y=\left\{(z, w) \in \mathbf{C}^{k} \times \mathbf{C}^{n}: z \in \bar{G}, p_{z}(w) \leq 1\right\}$, then $Y$ is the polynomial hull of $X$, provided $\bar{G}$ itself is polynomially convex. The theorem implies some regularity of the surface of $Y$ (in the direction of $w$ ) under similar assumption for $X$. A stronger result will be given in [21].

Proof (sketch). Whether $p_{z}(\cdot)$ are norms or quasinorms, part (b) follows from (a) in the same way as it was observed in the proof of Theorem 2.12(a). We omit further details.

(a) We prove the $P_{Q}^{\mathrm{co}}$ and $P_{Q}^{\mathrm{psh}}$ cases simultaneously. Let $W(z)=\{x \in$ $\left.\mathbf{C}^{n}: p_{z}(x) \leq 1\right\}, z \in \bar{G}$. Fix $\alpha>0$ and $h \in \mathbf{C}^{n}$, and denote $u(z, x)=$ $p_{z}(\alpha x+h)^{2}+p_{z}(\alpha x-h)^{2}$ and $v(z)=\max \{u(z, x): x \in W(z)\}$. By (6.1), $u \in P_{Q}^{\text {co }}\left(\right.$ resp. $\left.P_{Q}^{\text {psh }}\right)$, and since $\left\{\mathbf{C}^{n}, p_{z}\right\}_{z \in \bar{G}}$ is a $P_{Q}^{\text {co }}$ - (resp. $P_{Q}^{\text {psh }}$-) interpolation family, $\operatorname{gr}(W \mid G)$ has local maximum property with respect to functions of class $P_{Q}^{\text {co }}\left(\right.$ or $\left.P_{Q}^{\text {psh }}\right)$ (cf. [18, Theorem 2.3]), and so $\max v|\bar{G} \leq \max v| \partial G$ (cf. also [18, Proposition 1.3]). By (6.4), $v(\zeta) \leq 2 \alpha^{2}+K|h|^{2}$ for $\zeta \in \partial G$, and so $v(z) \leq \alpha^{2}+K|h|^{2}, z \in G$. Thus, if $p_{z}(x)=1$, then $p_{z}(\alpha x+h)^{2}+p_{z}(\alpha x-h)^{2} \leq$ $2 p_{z}(\partial x)^{2}+K|h|^{2}$. Since $\alpha$ and $h$ were arbitrary, the result follows. Q.E.D.

\section{CONCLUDING REMARKS}

All results of this paper remain true in the context of harmonic interpolation on complex homogeneous spaces, i.e. for $P_{Q}^{\text {psh }}$-interpolation, where $Q$ is the class of all usc functions subharmonic with respect to an invariant Laplacian on a complex homogeneous space (cf. $[18, \S \S 8.1,8.4])$. They hold, in particular, for the $M$-harmonic interpolation, which corresponds to $Q=$ the class of all $M$-subharmonic functions on the unit ball of $\mathbf{C}^{k}$ (cf. [18, §8.3]). Although all the proofs given above can be easily adapted to the more general setting, for the sake of simplicity of exposition, we have formulated our results only in the Euclidean case.

In the context of complex information over a simply connected planar domain, R. Rochberg [23] studied how the interpolating norm varies with the parameter $z$. It would be worthwhile to generalize his results to harmonic interpolation in several dimensions, and we believe that the present paper might play a helpful role in this respect. 


\section{REFERENCES}

1. H. Alexander and J. Wermer, Polynomial hulls with convex fibers, Math. Ann. 271 (1985), 99-109.

2. R. Coifman, M. Cwikel, R. Rochberg, Y. Sagher and G. Weiss, The complex method for interpolation of operators acting on families of Banach spaces, Lecture Notes in Math., vol. 779, Springer-Verlag, Berlin and New York, 1980, pp. 123-153.

3. _- Complex interpolation for families of Banach spaces, Proc. Sympos. Pure Math., vol. 35, part 2, Amer. Math. Soc., Providence, R.I., 1979, pp. 269-282.

4. __ A theory of complex interpolation for families of Banach spaces, Adv. in Math. 33 (1982), 203-229.

5. R. Coifman and S. Semmes, Interpolation of Banach spaces and nonlinear Dirichlet problems.

6. M. M. Day, Normed linear spaces, 3rd ed., Springer-Verlag, New York, 1973.

7. R. L. Hunt and J. J. Murray, q-plurisubharmonic functions and a generalized Dirichlet problem, Michigan Math. J. 25 (1978), 299-316.

8. J. Lindenstrauss and L. Tzafriri, Classical Banach spaces. II, Springer-Verlag, New York, 1979.

9. R. Rochberg, Interpolation of Banach spaces and negatively curved vector bundles, Pacific J. Math. 110 (1984), 355-376.

10. _ The work of Coifman and Semmes on complex interpolation, several complex variables and PDEs, U.S.-Swedish Seminar on Function Spaces and Applications, Lund, June 1986, Lecture Notes in Math., vol. 1302, Springer-Verlag, New York, 1988.

11. Z. Slodkowski, Analytic set-valued functions and spectra, Math. Ann. 256 (1981), 363-386.

12. _ The Bremermann-Dirichlet problem for q-plurisubharmonic functions, Ann. Scuola Norm. Sup. Pisa Cl. Sci. (4) 11 (1984), 303-326.

13. _- Analytic multifunctions, pseudoconvex domains and uniform algebras, Contemp. Math. 32 (1984), 243-258.

14. _ Local maximum property and q-plurisubharmonic functions in uniform algebras, J. Math. Anal. Appl. 115 (1986), 105-130.

15. An analytic set-valued selection and its applications to the corona theorem, to polynomial hulls and joint spectra, Trans. Amer. Math. Soc. 294 (1986), 367-377.

16. __ On bounded analytic functions in finitely connected domains, Trans. Amer. Math. Soc. 300 (1987), 721-736.

17. __ Pseudoconvex classes of functions. I. Pseudoconcave and pseudoconvex sets, Pacific J. Math. 134 (1988), 343-376.

18. __ Complex interpolation of normed and quasinormed spaces in several dimensions. I, Trans. Amer. Math. Soc. 308 (1988), 685-711.

19. __ Pseudoconvex classes of functions. III. Characterization of dual pseudoconvex classes on complex homogeneous spaces, Trans. Amer. Math. Soc. 309 (1988), 165-189.

20. __ Pseudoconvex classes of functions. II, Pacific J. Math. (to appear).

21. Complex interpolation of normed and quasinormed spaces in several dimensions. III, Trans. Amer. Math. Soc. (to appear).

22. J. Wermer, letter.

23. R. Rochberg, Function theoretic results for complex interpolation families of Banach spaces, Trans. Amer. Math. Soc. 284 (1984), 745-758.

24. M. Cwikel and S. Reisner, Interpolation of uniformly convex Banach spaces, Proc. Amer. Math. Soc. 84 (1982), 555-559.

25. M. Vignati, Complex interpolation and convexity, Proc. Amer. Math. Soc. 99 (1987), 705-711.

Department of Mathematics, University of Illinois at Chicago, Chicago, Illinois 60680 